\title{
ON IDENTIFYING MAGNETIZED ANOMALIES USING GEOMAGNETIC MONITORING II. A MAGNETOHYDRODYNAMIC MODEL
}

\author{
YOUJUN DENG, JINHONG LI, AND HONGYU LIU
}

\begin{abstract}
This paper is a continuation and an extension of our recent work 13 on the identification of magnetized anomalies using geomagnetic monitoring, which aims to establish a rigorous mathematical theory for the geomagnetic detection technology. Suppose a collection of magnetized anomalies is presented in the shell of the Earth. By monitoring the variation of the magnetic field of the Earth due to the presence of the anomalies, we establish sufficient conditions for the unique recovery of those unknown anomalies. In [13], the geomagnetic model was described by a linear Maxwell system. In this paper, we consider a much more sophisticated and complicated magnetohydrodynamic model, which stems from the widely accepted dynamo theory of geomagnetics.
\end{abstract}

Keywords: Magnetohydrodynamics, geomagnetic monitoring, magnetic anomaly detection, unique recovery

2010 Mathematics Subject Classification: 35Q60, 35J05, 31B10, 35R30, 78A40

\section{INTRODUCTION}

1.1. Background on the dynamo theory of geomagnetics. The dynamo theory proposes a mechanism such that a rotating, convecting and electrically conducting fluid can maintain the magnetic field of a celestial body. Earth's magnetic field has been interpreted by the dynamo theory through a magnetohydrodynamic (MHD) model $6,9,16,18,21,22,24$ [27]. Following the discussion in [8, 10, 26, 27, we briefly introduce the MHD system for our study. It is widely accepted that the Earth is of a core-shell structure. The dynamo mechanism states that the convecting currents of fluid metal in the Earth's outer core, driven by heat flow from the outer core, organized into rolls by the Coriolis force, create circulating electric currents, which generate the magnetic field. To describe this physical process, there are three basic partial differential equations including the Navier-Stokes equations, the heat equation and the Maxwell equations, and they are coupled together to form the MHD system.

Let $\Sigma_{i}, \Sigma_{o}$ and $\Sigma_{s}$ be bounded simply-connected $C^{2}$ domains in $\mathbb{R}^{3}$, respectively, signifying the inner core, outer core and shell of the Earth. $\Sigma_{c}:=\Sigma_{o} \cup \overline{\Sigma_{i}}$ and $\Sigma:=\Sigma_{s} \cup \overline{\Sigma_{c}}$, are also simply-connected $C^{2}$ domains, which denote, respectively, the Earth's core and the Earth. $\mathbb{R}^{3} \backslash \bar{\Sigma}$ is the outer space of the Earth. Let $\mathbf{u}(\mathbf{x}, t)$ and $p(\mathbf{x}, t),(\mathbf{x}, t) \in \Sigma_{o} \times \mathbb{R}_{+}$, respectively denote the velocity field and pressure of the fluid metal in the outer core. Throughout, $\mathbf{x} \in \mathbb{R}^{3}$ represents the position vector and $t$ represents the time variable. Let $\Theta(\mathbf{x}, t),(\mathbf{x}, t) \in \Sigma_{o} \times \mathbb{R}_{+}$, signify the deviation of the temperature from its static distribution of the Earth's outer core. Finally, we let $\mathbf{B}(\mathbf{x}, t)$ and $\mathcal{H}(\mathbf{x}, t),(\mathbf{x}, t) \in \mathbb{R}^{3} \backslash \overline{\Sigma_{i}} \times \mathbb{R}_{+}$ respectively, be the induced magnetic field and the intensity of the magnetic field. There holds $\mathbf{B}(\mathbf{x}, t)=\mu(\mathbf{x}) \mathcal{H}(\mathbf{x}, t), \mathbf{x} \in \mathbb{R}^{3} \backslash \overline{\Sigma_{i}}$, where $\mu(\mathbf{x})$ is the magnetic permeability of the medium. 
The motion of the fluid metal in the outer core is described by the following Navier-Stokes equations

$$
\dot{\mathbf{u}}+\mathbf{u} \cdot \nabla \mathbf{u}-\eta \Delta \mathbf{u}+2 \widetilde{\omega} \times \mathbf{u}+\frac{1}{\varrho} \nabla p=\gamma \alpha \Theta \mathbf{x}+\frac{1}{\varrho \mu}(\nabla \times \mathbf{B}) \times \mathbf{B}, \quad(\mathbf{x}, t) \in \Sigma_{o} \times \mathbb{R}_{+},
$$

where and also in what follows, the overdot signifies the partial derivative with respect to the time variable $t$. Furthermore, the incompressibility of the fluid yields that

$$
\nabla \cdot \mathbf{u}(\mathbf{x}, t)=0, \quad(\mathbf{x}, t) \in \Sigma_{o} \times \mathbb{R}_{+} .
$$

The temperature distribution $\Theta(\mathbf{x}, t)$ satisfies the following heat equation

$$
\dot{\Theta}+\mathbf{u} \cdot \nabla \Theta=\kappa \Delta \Theta+\beta \mathbf{u} \cdot \mathbf{x}, \quad(\mathbf{x}, t) \in \Sigma_{o} \times \mathbb{R}_{+} .
$$

The physical meaning of the coefficient parameters in (1.1) and (1.3) are given as follows. $\varrho$ is the density, $\kappa$ is the thermal diffusivity, $\alpha$ is the thermal expansion coefficient, $\eta$ is the kinematic viscosity and $\lambda$ is the magnetic diffusivity. There holds,

$$
\lambda=\frac{1}{\mu \sigma},
$$

where $\mu$ and $\sigma$ are respectively the magnetic permeability and electric conductivity. $\widetilde{\omega}$ signifies the uniform angular velocity of the rotation of the Earth's core. $\gamma$ is a positive constant which fulfils $\mathbf{g}=-\gamma \mathbf{x}$, where $\mathbf{g}$ is the gravitational field of the Earth. The parameter $\beta$ is a positive constant representing the uniformly distributed heat source. To complete the description, one needs to impose suitable boundary and initial conditions for the two partial differential equations (1.1) and (1.3). There are two types of widely used boundary conditions. In this paper, we assume that the inner- and outer-bounding surfaces are nonslip and impenetrable, namely,

$$
\mathbf{u}(\mathbf{x}, t)=0, \quad(\mathbf{x}, t) \in \partial \Sigma_{o} \times \mathbb{R}_{+} .
$$

One may also assume that the velocity on the boundary, $\partial \Sigma_{o}$, is stress-free and impenetrable. The temperature boundary condition usually does not play a major role, and one may assume that the heat does not convect in the region outside the outer core, i.e.,

$$
\frac{\partial \Theta(\mathbf{x}, t)}{\partial \nu}=0 . \quad(\mathbf{x}, t) \in \partial \Sigma_{o} \times \mathbb{R}_{+},
$$

where $\nu$ denotes the exterior unit normal vector to $\partial \Sigma_{o}$. The initial conditions are prescribed as follows,

$$
\mathbf{u}(\mathbf{x}, 0)=\mathbf{f}(\mathbf{x}), \quad \Theta(\mathbf{x}, 0)=T(\mathbf{x}), \quad \mathbf{x} \in \Sigma_{o} .
$$

Here, we would like to emphasize that the boundary and initial conditions shall not play an essential role in our study of the inverse problem on the geomagnetic detection as long as the corresponding forward problem is well-posed. We shall further remark this point in our subsequent discussion.

The induced magnetic field is governed by the following exterior problem associated with a Maxwell system,

$$
\begin{cases}\dot{\mathcal{H}}(\mathbf{x}, t)=\nabla \times(\mathbf{u}(\mathbf{x}, t) \times \mathcal{H}(\mathbf{x}, t))-\lambda_{o}(\mathbf{x}) \nabla \times \nabla \times \mathcal{H}(\mathbf{x}, t), & (\mathbf{x}, t) \in \Sigma_{o} \times \mathbb{R}_{+}, \\ \dot{\mathcal{H}}(\mathbf{x}, t)=-\lambda_{s}(\mathbf{x}) \nabla \times \nabla \times \mathcal{H}(\mathbf{x}, t), & (\mathbf{x}, t) \in \Sigma_{s} \times \mathbb{R}_{+}, \\ \nabla \times \nabla \times \mathcal{H}(\mathbf{x}, t)=0, & (\mathbf{x}, t) \in\left(\mathbb{R}^{3} \backslash \bar{\Sigma}\right) \times \mathbb{R}_{+}, \\ \nabla \cdot(\mu(\mathbf{x}) \mathcal{H}(\mathbf{x}, t))=0, \quad(\mathbf{x}, t) \in\left(\mathbb{R}^{3} \backslash \overline{\Sigma_{i}}\right) \times \mathbb{R}_{+} ; \mathcal{H}(\mathbf{x}, 0)=\mathbf{h}(\mathbf{x}) \chi\left(\Sigma_{o}\right), & \mathbf{x} \in \mathbb{R}^{3} \backslash \overline{\Sigma_{i}}, \\ \nu \times \mathcal{H}(\mathbf{x}, t)=0, \quad(\mathbf{x}, t) \in \partial \Sigma_{i} \times \mathbb{R}_{+} ; \quad \mathcal{H}(\mathbf{x}, t)=\mathcal{O}\left(\|\mathbf{x}\|^{-2}\right) \text { as }\|\mathbf{x}\| \rightarrow \infty,\end{cases}
$$


where $\lambda_{o}$ and $\lambda_{s}$ are respectively the magnetic diffusivities in the outer core and shell of the Earth. In (1.8), we impose a perfect magnetic conducting (PMC) condition on the inner boundary $\Sigma_{i}$. As emphasized earlier, this boundary condition shall not play an essential role in our subsequent inverse problem study as long as the MHD system (1.1)-(1.8) is wellposed in the sense that shall be prescribed later. It can be replaced by any other suitable boundary condition depending on the availability of the physical property of the inner core. In what follows, for expositional convenience, we refer to $\mathcal{H}$ as the magnetic field.

1.2. Mathematical formulation of the geomagnetic detection. We present the mathematical formulation of the geomagnetic detection. Let $\mu$ and $\sigma$ be real-valued $L^{\infty}$ functions in $\mathbb{R}^{3} \backslash \overline{\Sigma_{i}}$, such that $\mu$ is positive and $\sigma$ is nonnegative. Let $\mu_{0}$ denote the permeability of the uniformly homogeneous outer space $\mathbb{R}^{3} \backslash \bar{\Sigma}$. The material distribution in $\mathbb{R}^{3} \backslash \overline{\Sigma_{i}}$ is described by

$$
\sigma(\mathbf{x})=\sigma_{c}(\mathbf{x}) \chi\left(\Sigma_{o}\right)+\sigma_{s}(\mathbf{x}) \chi\left(\Sigma_{s}\right), \quad \mu(\mathbf{x})=\left(\mu_{c}(\mathbf{x})-\mu_{0}\right) \chi\left(\Sigma_{o}\right)+\mu_{0}, \quad \mathbf{x} \in \mathbb{R}^{3} \backslash \overline{\Sigma_{i}},
$$

where and also in what follows, $\chi$ denotes the characteristic function. By (1.9), we know that the mediums in the outer core and the shell of the Earth are respectively characterized by $\left(\Sigma_{o} ; \mu_{c}, \sigma_{c}\right)$ and $\left(\Sigma_{s} ; \mu_{0}, \sigma_{s}\right)$. Define by $\lambda$ the magnetic diffusivity in the Earth's interior, then it satisfies

$$
\lambda(\mathbf{x})=\lambda_{c}(\mathbf{x}) \chi\left(\Sigma_{o}\right)+\lambda_{s}(\mathbf{x}) \chi\left(\Sigma_{s}\right),
$$

where $\lambda_{c}(\mathbf{x})=\frac{1}{\mu_{c} \sigma_{c}}$ and $\lambda_{s}(\mathbf{x})=\frac{1}{\mu_{0} \sigma_{s}}$. Henceforth, we let $\mathbf{u}_{0}, p_{0}, \Theta_{0}$, and $\mathcal{H}_{0}$, respectively, denote the velocity field, pressure function, temperature distribution and magnetic field of the MHD system (1.1)-(1.8) associated with the medium configuration in (1.9) and (1.10).

Next we suppose that a collection of magnetized anomalies presented in the shell of the Earth. Let $D_{l}, l=1,2, \ldots, l_{0}$, denote the magnetized anomalies, where $D_{l}, 1 \leq l \leq l_{0}$ are simply-connected $C^{2}$ domains such that the corresponding material parameters are given by $\mu_{l}$ and $\sigma_{l}$. It is assumed that $\mu_{l}$ and $\sigma_{l}$ are all positive constants with $\mu_{l} \neq \mu_{0}, 1 \leq l \leq l_{0}$. With the presence of the magnetized anomalies $\left(D_{l} ; \mu_{l}, \sigma_{l}\right), l=1,2, \ldots, l_{0}$, in the shell of the Earth, the medium configuration in $\mathbb{R}^{3} \backslash \overline{\Sigma_{i}}$ is then described by

$$
\begin{aligned}
& \tilde{\sigma}(\mathbf{x})=\sigma_{c}(\mathbf{x}) \chi\left(\Sigma_{o}\right)+\sigma_{s}(\mathbf{x}) \chi\left(\Sigma_{s}\right)+\sum_{l=1}^{l_{0}} \sigma_{l} \chi\left(D_{l}\right), \\
& \tilde{\mu}(\mathbf{x})=\left(\mu_{c}(\mathbf{x})-\mu_{0}\right) \chi\left(\Sigma_{o}\right)+\sum_{l=1}^{l_{0}}\left(\mu_{l}-\mu_{0}\right) \chi\left(D_{l}\right)+\mu_{0},
\end{aligned}
$$

Accordingly, the magnetic diffusivity $\tilde{\lambda}$ is given by

$$
\tilde{\lambda}(\mathbf{x})=\lambda_{c}(\mathbf{x}) \chi\left(\Sigma_{o}\right)+\lambda_{s}(\mathbf{x}) \chi\left(\Sigma_{s} \backslash \bigcup_{l=1}^{\overline{l_{0}} D_{l}}\right)+\sum_{l=1}^{l_{0}} \lambda_{l}(x) \chi\left(D_{l}\right),
$$

where $\lambda_{l}(\mathbf{x})=\frac{1}{\mu_{l} \sigma_{l}}, l=1,2, \ldots, l_{0}$. Let $\widetilde{\mathbf{u}}, \widetilde{p}, \widetilde{\Theta}$, and $\widetilde{\mathcal{H}}$, respectively, denote the velocity field, pressure function, temperature distribution and magnetic field of the MHD system (1.1) - (1.8) associated with the medium configuration in (1.11) and (1.12).

Let $\widetilde{\Gamma}$ be a simply-connected and analytic surface which encloses $\Sigma$, and let $\Gamma$ be an open patch of $\widetilde{\Gamma}$. In the current article, we are mainly concerned with the following inverse problem,

$$
\left.\left(\tilde{\mathcal{H}}(\mathbf{x}, t)-\mathcal{H}_{0}(\mathbf{x}, t)\right)\right|_{(\mathbf{x}, t) \in \Gamma \times \mathbb{R}_{+}} \longrightarrow \bigcup_{l=1}^{l_{0}}\left(D_{l} ; \mu_{l}, \sigma_{l}\right) .
$$


That is, we make use the variation of the geomagnetic field due to the presence of the magnetized anomalies in the shell of the Earth to recover the locations as well as the material parameters of the anomalies. For simplicity, one may take $\Gamma$ to be an open patch of $\partial B_{R}$ with $B_{R}$ a sufficiently large central ball containing $\Sigma$. Several remarks are in order regarding the magnetic anomaly detection problem described above.

First, from a practical point of view, the geomagnetic configuration in the core of the Earth, namely the fluid metal, should not be assumed to be a-priori known. In fact, the movement of the fluid metal in the outer core is to generate the source input for the Maxwell system (1.8). On the other hand, the measurement data $\mathcal{H}_{0}$ clearly encode the information of this generated source. This observation is critical for our subsequent unique recovery study of the geomagnetic detection. Second, the time interval for the measurement in (1.13) is $\mathbb{R}_{+}$. However, it can actually be replaced by a finite time-interval $\left[0, T_{0}\right]$ such that after $T_{0}$, the interrupted geomagnetic field due to the presence of the anomalies leaves away from the Earth. This is clearly a physically reasonable assumption. Third, the measurement surface $\Gamma$ is in a scale much smaller than the Earth, and we are mainly concerned with the region under $\Gamma$ such that the geomagnetic effect can reach $\Gamma$. Hence, it is unobjectionable to assume that the medium configuration in the rest part of the Earth is the same as that in the region under $\Gamma$. One may consider the submarine detection using the geomagnetic monitoring and it can be assumed that the magnetic diffusivity $\lambda_{s}$ is the one for the sea. Throughout the rest of the paper, we assume that $\lambda_{s}$ is a constant and it is known a-priori. Finally, it is noted that the anomalies are also in a size much smaller than that of the Earth.

According to our discussion above, it is sufficient for us to consider the inverse problem (1.13) associated with the magnetohydrodynamic Maxwell system (1.8) in $\mathbb{R}^{3} \backslash \overline{\Sigma_{c}}$, with the implicit use that the magnetic fields are generated by the outer core of the Earth through the MHD system (1.1)-(1.8). The well-posedness of the complicated nonlinear MHD system (1.1) -(1.8) is a challenging problem and a large amount literature is devoted to this topic. We shall not explore this topic in the current article and our focus is the geomagnetic detection problem. Nevertheless, we need the following technical assumption for our study on the magnetic fields $\mathcal{H}_{0}$ and $\widetilde{\mathcal{H}}$ in $\mathbb{R}^{3} \backslash \overline{\Sigma_{c}}$. Introduce the following temporal Fourier transform for $(\mathbf{x}, \omega) \in\left(\mathbb{R}^{3} \backslash \overline{\Sigma_{c}}\right) \times \mathbb{R}_{+}$,

$$
\mathbf{J}(\mathbf{x}, \omega)=\mathcal{F}_{t}(\mathcal{J}):=\frac{1}{2 \pi} \int_{0}^{\infty} \mathcal{J}(\mathbf{x}, t) e^{\mathrm{i} \omega t} d t, \quad \mathcal{J}=\mathcal{H}_{0} \quad \text { or } \quad \widetilde{\mathcal{H}}
$$

Throughout, we assume that the Fourier transform (1.14) exists for $(\mathbf{x}, \omega) \in\left(\mathbb{R}^{3} \backslash \overline{\Sigma_{c}}\right) \times$ $\left(0, \omega_{0}\right)$ with $\omega_{0} \in \mathbb{R}_{+}$such that $\mathbf{J}(\mathbf{x}, \omega) \in H_{\text {loc }}\left(\operatorname{curl}, \mathbb{R}^{3} \backslash \overline{\Sigma_{c}}\right)$. Here and also in what follows,

$$
H_{l o c}(\operatorname{curl}, X):=\left\{\left.U\right|_{\Omega} \in H(\operatorname{curl}, \Omega) \mid \Omega \text { is any bounded subdomain of } X\right\},
$$

and

$$
H(\operatorname{curl}, \Omega)=\left\{U \in\left(L^{2}(\Omega)\right)^{3} \mid \nabla \times U \in\left(L^{2}(\Omega)\right)^{3}\right\} .
$$

Set

$$
\mathbf{H}_{0}=\mathcal{F}_{t}\left(\mathcal{H}_{0}\right), \mathbf{H}=\mathcal{F}_{t}(\widetilde{\mathcal{H}})
$$

From the time-dependent system (1.8) as well as the description of the medium configurations in (1.9) $-(1.12)$ associated with $\mathcal{H}_{0}$ and $\widetilde{\mathcal{H}}$, respectively, one has by direct verifications the following two systems in the frequency-domain, respectively, for $\mathbf{H}_{0} \in H_{\text {loc }}\left(\operatorname{curl}, \mathbb{R}^{3} \backslash \overline{\Sigma_{c}}\right)$ 
and $\mathbf{H} \in H_{l o c}\left(\operatorname{curl}, \mathbb{R}^{3} \backslash \overline{\Sigma_{c}}\right)$ :

$$
\begin{cases}\nabla \times \nabla \times \mathbf{H}_{0}(\mathbf{x})-i \omega \lambda_{s}^{-1} \mathbf{H}_{0}(\mathbf{x})=0, & \mathbf{x} \in \Sigma_{s}, \\ \nabla \times \nabla \times \mathbf{H}_{0}(\mathbf{x})=0, & \mathbf{x} \in \mathbb{R}^{3} \backslash \bar{\Sigma}, \\ \nabla \cdot\left(\mu(\mathbf{x}) \mathbf{H}_{0}(\mathbf{x})\right)=0, & \mathbf{x} \in \mathbb{R}^{3} \backslash \overline{\Sigma_{c}} \\ \mathbf{H}_{0}=\mathcal{O}\left(\|\mathbf{x}\|^{-2}\right), & \|\mathbf{x}\| \rightarrow \infty\end{cases}
$$

and

$$
\begin{cases}\nabla \times \nabla \times \mathbf{H}(\mathbf{x})-i \omega \lambda_{s}^{-1} \mathbf{H}(\mathbf{x})=0, & \mathbf{x} \in \Sigma_{s} \backslash \overline{\bigcup_{l=1}^{l_{0}} D_{l}}, \\ \nabla \times \nabla \times \mathbf{H}(\mathbf{x})-i \omega \lambda_{l}^{-1} \mathbf{H}(\mathbf{x})=0, & \mathbf{x} \in D_{l}, \quad l=1,2, \ldots, l_{0}, \\ \nabla \times \nabla \times \mathbf{H}(\mathbf{x})=0, & \mathbf{x} \in \mathbb{R}^{3} \backslash \bar{\Sigma} \\ \nabla \cdot(\tilde{\mu}(\mathbf{x}) \mathbf{H}(\mathbf{x}))=0, & \mathbf{x} \in \mathbb{R}^{3} \backslash \overline{\Sigma_{c}} \\ \mathbf{H}=\mathcal{O}\left(\|\mathbf{x}\|^{-2}\right), & \|\mathbf{x}\| \rightarrow \infty\end{cases}
$$

Based on the above Fourier reformulation, the inverse problem (1.13) can be recast as

$$
\left.\left(\mathbf{H}(\mathbf{x}, \omega)-\mathbf{H}_{0}(\mathbf{x}, \omega)\right)\right|_{(\mathbf{x}, \omega) \in \Gamma \times\left(0, \omega_{0}\right)} \longrightarrow \bigcup_{l=1}^{l_{0}}\left(D_{l} ; \mu_{l}, \sigma_{l}\right) .
$$

We shall follow the general strategy developed in [13] to tackle the inverse problem (1.18). That is, we mainly make use of the static parts of the magnetic fields for the recovery of the inverse problem (1.18). To that end, one needs to derive the low-frequency asymptotics of the magnetic fields $\mathbf{H}_{0}$ and $\mathbf{H}$. However, there are several new challenges as well as the corresponding novel technical developments in the current setup of study. The geomagnetic model is a linear Maxwell system in [13, and hence the low-frequency asymptotics therein were conducted globally for the whole system. However, the MHD system is a coupled nonlinear system and similar global low-frequency asymptotics of the Maxwell system (1.17) in coupling with the whole MHD system shall be fraught with various difficulties. A critical new technical ingredient in the present work is that we treat the measurement data of $\mathbf{H}$ on $\Gamma$ as source inputs for the Maxwell system (1.17), and one actually can continue the data up to the boundary of the core, namely $\partial \Sigma_{c}$. Indeed, by analytic continuation, one can first analytically extend the data on $\Gamma$ to the closed surface $\widetilde{\Gamma}$, and then by solving the homogeneous Maxwell system in the outer space, one can further extend the data to $\mathbb{R}^{3} \backslash \bar{\Sigma}$, in particular to the boundary of the Earth $\partial \Sigma$. Since $\lambda_{s}$ is known in $\Sigma_{s}$, one can then continue the Cauchy data on $\partial \Sigma$ to $\partial \Sigma_{o}$ through the Maxwell system in $\Sigma_{s} \backslash \overline{\bigcup_{l=1}^{l_{0}} D_{l}}$. By incorporating such a critical data continuation into our argument, we can then perform the low-frequency asymptotics locally, that is, outside the core of the Earth. We believe this kind of strategy could be potentially used to deal with other inverse problems associated with certain coupled PDEs in different contexts. Furthermore, we would like to point out that in addition to this "localization" issue, the Maxwell system (1.17) is of a different mathematical nature from that considered in [13]. After deriving the low-frequency asymptotics, we then further linearize the nonlinear inverse problem (1.18) with respect to the small-size of the magnetized anomalies and finally arrive at the unique recovery results.

The rest of the paper is organized as follows. In Sections 2 and 3, we derive the lowasymptotic expansions of the magnetic fields as well as the leading-order terms of the static magnetic fields with respect to the small-size of the magnetized anomalies. In Section 4, we establish the unique recovery results for the geomagnetic detection problem. 


\section{INTEGRAL REPRESENTATION AND ASYMPTOTIC ANALYSIS}

We make essential use of the layer potential theory in our study. In what follows, we first collect some preliminary knowledge on the layer potential theory. Then we conduct extensive asymptotic analysis in deriving the low-frequency asymptotic expansions of the magnetic fields as well as the leading-order terms of the static magnetic fields with respect to the small-size of the magnetized anomalies associated with the Maxwell systems (1.16) and (1.17).

2.1. Layer potentials. For a bounded $C^{2}$ domain $B \subset \mathbb{R}^{3}$, we first introduce some function spaces on the boundary $\partial B$ for the subsequent use. Let $\nabla_{\partial B}$. denote the surface divergence. Denote by $L_{T}^{2}(\partial B):=\left\{\Phi \in L^{2}(\partial B)^{3}, \nu \cdot \Phi=0\right\}$. Let $H^{s}(\partial B)$ be the usual Sobolev space of order $s \in \mathbb{R}$ on $\partial B$. Set

$$
\begin{aligned}
\mathrm{TH}(\operatorname{div}, \partial B) & :=\left\{\Phi \in L_{T}^{2}(\partial B): \nabla_{\partial B} \cdot \Phi \in L^{2}(\partial B)\right\}, \\
\mathrm{TH}(\operatorname{curl}, \partial B) & :=\left\{\Phi \in L_{T}^{2}(\partial B): \nabla_{\partial B} \cdot(\Phi \times \nu) \in L^{2}(\partial B)\right\},
\end{aligned}
$$

endowed with the norms

$$
\begin{aligned}
& \|\Phi\|_{\mathrm{TH}(\operatorname{div}, \partial B)}=\|\Phi\|_{L^{2}(\partial B)}+\left\|\nabla_{\partial B} \cdot \Phi\right\|_{L^{2}(\partial B)}, \\
& \|\Phi\|_{\mathrm{TH}(\operatorname{curl}, \partial B)}=\|\Phi\|_{L^{2}(\partial B)}+\left\|\nabla_{\partial B} \cdot(\Phi \times \nu)\right\|_{L^{2}(\partial B)},
\end{aligned}
$$

respectively. Let $\Gamma_{k}$ be the fundamental solution to the PDO $\left(\Delta+k^{2}\right)$, which is given by

$$
\Gamma_{k}(\mathbf{x})=-\frac{e^{i k\|\mathbf{x}\|}}{4 \pi\|\mathbf{x}\|}, \quad \mathbf{x} \in \mathbb{R}^{3} \text { and } \mathbf{x} \neq \mathbf{0}
$$

For any bounded domain $B \subset \mathbb{R}^{3}$, we denote by $\mathcal{S}_{B}^{k}: H^{-1 / 2}(\partial B) \rightarrow H^{1}\left(\mathbb{R}^{3} \backslash \partial B\right)$ the single layer potential operator given by

$$
\mathcal{S}_{B}^{k}[\phi](\mathbf{x}):=\int_{\partial B} \Gamma_{k}(\mathbf{x}-\mathbf{y}) \phi(\mathbf{y}) d s_{\mathbf{y}}
$$

and $\mathcal{K}_{B}^{k}: H^{1 / 2}(\partial B) \rightarrow H^{1 / 2}(\partial B)$ the Neumann-Poincaré operator

$$
\mathcal{K}_{B}^{k}[\phi](\mathbf{x}):=\text { p.v. } \quad \int_{\partial B} \frac{\partial \Gamma_{k}(\mathbf{x}-\mathbf{y})}{\partial \nu_{y}} \phi(\mathbf{y}) d s_{\mathbf{y}},
$$

where p.v. stands for the Cauchy principle value. In (2.3) and also in what follows, unless otherwise specified, $\nu$ signifies the exterior unit normal vector to the boundary of the concerned domain. It is known that the single layer potential operator $\mathcal{S}_{B}^{k}$ satisfies the following trace formula

$$
\left.\frac{\partial}{\partial \nu} \mathcal{S}_{B}^{k}[\phi]\right|_{ \pm}=\left( \pm \frac{1}{2} I+\left(\mathcal{K}_{B}^{k}\right)^{*}\right)[\phi] \quad \text { on } \partial B
$$

where $\left(\mathcal{K}_{B}^{k}\right)^{*}$ is the adjoint operator of $\mathcal{K}_{B}^{k}$.

For a density function $\Phi \in \mathrm{TH}(\operatorname{div}, \partial B)$, we define the vectorial single layer potential by

$$
\mathcal{A}_{B}^{k}[\Phi](\mathbf{x}):=\int_{\partial B} \Gamma_{k}(\mathbf{x}-\mathbf{y}) \Phi(\mathbf{y}) d s_{\mathbf{y}}, \quad \mathbf{x} \in \mathbb{R}^{3} \backslash \partial B .
$$

It is known that $\nabla \times \mathcal{A}_{B}^{k}$ satisfies the following jump formula

$$
\nu \times \nabla \times\left.\mathcal{A}_{B}^{k}[\Phi]\right|_{ \pm}=\mp \frac{\Phi}{2}+\mathcal{M}_{B}^{k}[\Phi] \quad \text { on } \partial B
$$

where

$$
\forall \mathbf{x} \in \partial B, \quad \nu \times \nabla \times\left.\mathcal{A}_{B}^{k}[\Phi]\right|_{ \pm}(\mathbf{x})=\lim _{t \rightarrow 0^{+}} \nu \times \nabla \times \mathcal{A}_{B}^{k}[\Phi](\mathbf{x} \pm t \nu)
$$


and

$$
\mathcal{M}_{B}^{k}[\Phi](\mathbf{x})=\text { p.v. } \quad \nu \times \nabla \times \int_{\partial B} \Gamma_{k}(\mathbf{x}-\mathbf{y}) \Phi(\mathbf{y}) d s_{\mathbf{y}} .
$$

We also define $\mathcal{L}_{B}^{k}: L^{2}(\partial B) \rightarrow \mathrm{TH}(\operatorname{div}, \partial B)$ by

$$
\mathcal{L}_{B}^{k}[\varphi](\mathbf{x}):=\nu_{\mathbf{x}} \times \nabla \mathcal{S}_{B}^{k}[\varphi](\mathbf{x}),
$$

and $\mathcal{N}_{B}^{k}: \mathrm{TH}(\operatorname{div}, \partial B) \rightarrow L^{2}(\partial B)$ by

$$
\mathcal{N}_{B}^{k}[\Phi](\mathbf{x}):=\nu_{\mathbf{x}} \cdot\left(\nabla \times \mathcal{A}_{B}^{k}[\Phi](\mathbf{x})\right) .
$$

It is mentioned that $\frac{I}{2} \pm \mathcal{M}_{B}^{k}$ is invertible on $\mathrm{TH}(\operatorname{div}, \partial \mathrm{B})$ when $k$ is sufficiently small (see e.g., 1, 23). In the following, if $k=0$, we formally set $\Gamma_{k}$ introduced in (2.1) to be $\Gamma_{0}=-1 /(4 \pi\|\mathbf{x}\|)$, and the other integral operators introduced above can also be formally defined when $k=0$.

Throughout the rest of the paper, we set

$$
k^{2}:=-i \frac{\omega}{\lambda}, \quad \Re k \geq 0,
$$

where $\lambda$ is a constant magnetic diffusivity. $k_{s}^{2}, k_{c}^{2}$ and $k_{l}^{2}, l=1,2, \ldots, l_{0}$ are defined similarly to $k^{2}$ by replacing $\lambda$ with $\lambda_{s}, \lambda_{c}$ and $\lambda_{l}, l=1,2, \ldots, l_{0}$, respectively.

2.2. Low-frequency asymptotic expansions of the magnetic fields. In this subsection, we give the low-frequency asymptotic expansions of the magnetic fields $\mathbf{H}_{0}$ and $\mathbf{H}$ associated with (1.16) and (1.17), respectively. We would like to mention in passing some related literature on the low-frequency asymptotic analysis of the Maxwell system [3, 5, 11, 14, 17]. We first deal with $\mathbf{H}_{0}$, which can be represented by the following integral ansatz,

$$
\mathbf{H}_{0}= \begin{cases}\nabla \times \mathcal{A}_{\Sigma_{c}}^{0}\left[\Phi_{c}\right]+\nabla \times \mathcal{A}_{\Sigma}^{0}\left[\Phi_{s}\right]+\nabla \times \nabla \times \mathcal{A}_{\Sigma}^{0}\left[\nabla_{\partial \Sigma} \varphi_{s}\right] & \text { in } \mathbb{R}^{3} \backslash \bar{\Sigma}, \\ \nabla \times \mathcal{A}_{\Sigma_{c}}^{k_{s}}\left[\Phi_{c}\right]+\nabla \times \mathcal{A}_{\Sigma}^{k_{s}}\left[\Phi_{s}\right]+\nabla \times \nabla \times \mathcal{A}_{\Sigma}^{k_{s}}\left[\nabla_{\partial \Sigma} \varphi_{s}\right] & \text { in } \Sigma_{s},\end{cases}
$$

where $\left(\Phi_{c}, \Phi_{s}, \varphi_{s}\right) \in \mathrm{TH}\left(\operatorname{div}, \partial \Sigma_{\mathrm{c}}\right) \times \mathrm{TH}(\operatorname{div}, \partial \Sigma) \times \mathrm{H}^{2}(\partial \Sigma)$. By the continuous property of $\nu \times \mathbf{H}_{0}$ across the boundary $\partial \Sigma_{c}$ and $\partial \Sigma$, one has

$\nu \times \nabla \times\left.\mathcal{A}_{\Sigma_{c}}^{k_{s}}\left[\Phi_{c}\right]\right|_{\partial \Sigma_{c}} ^{+}+\nu \times \nabla \times\left.\mathcal{A}_{\Sigma}^{k_{s}}\left[\Phi_{s}\right]\right|_{\partial \Sigma_{c}} ^{+}+\nu \times \nabla \times \nabla \times\left.\mathcal{A}_{\Sigma}^{k_{s}}\left[\nabla_{\partial \Sigma} \varphi_{s}\right]\right|_{\partial \Sigma_{c}} ^{+}=\nu \times\left.\mathbf{H}_{0}\right|_{\partial \Sigma_{c}} ^{+}$,

and

$$
\begin{aligned}
& \nu \times \nabla \times\left.\mathcal{A}_{\Sigma_{c}}^{k_{s}}\left[\Phi_{c}\right]\right|_{\partial \Sigma} ^{-}+\nu \times \nabla \times\left.\mathcal{A}_{\Sigma}^{k_{s}}\left[\Phi_{s}\right]\right|_{\partial \Sigma} ^{-}+\nu \times \nabla \times \nabla \times\left.\mathcal{A}_{\Sigma}^{k_{s}}\left[\nabla_{\partial \Sigma} \varphi_{s}\right]\right|_{\partial \Sigma} ^{-} \\
= & \nu \times \nabla \times\left.\mathcal{A}_{\Sigma_{c}}^{0}\left[\Phi_{c}\right]\right|_{\partial \Sigma} ^{+}+\nu \times \nabla \times\left.\mathcal{A}_{\Sigma}^{0}\left[\Phi_{s}\right]\right|_{\partial \Sigma} ^{+}+\nu \times \nabla \times \nabla \times\left.\mathcal{A}_{\Sigma}^{0}\left[\nabla_{\partial \Sigma} \varphi_{s}\right]\right|_{\partial \Sigma} ^{+},
\end{aligned}
$$

On the other hand, by the continuous of $\nu \cdot \mu \mathbf{H}_{0}$ ((see Lemma 3.3 in [15])) across the boundary $\partial \Sigma$, one further has

$$
\begin{aligned}
& \left.\nu \cdot\left(\nabla \times \mathcal{A}_{\Sigma_{c}}^{k_{s}}\left[\Phi_{c}\right]\right)\right|_{\partial \Sigma} ^{-}+\left.\nu \cdot\left(\nabla \times \mathcal{A}_{\Sigma}^{k_{s}}\left[\Phi_{s}\right]\right)\right|_{\partial \Sigma} ^{-}+\nu \cdot \nabla \times \nabla \times\left.\mathcal{A}_{\Sigma}^{k_{s}}\left[\nabla_{\partial \Sigma} \varphi_{s}\right]\right|_{\partial \Sigma} ^{-} \\
= & \left.\nu \cdot\left(\nabla \times \mathcal{A}_{\Sigma_{c}}^{0}\left[\Phi_{c}\right]\right)\right|_{\partial \Sigma} ^{+}+\left.\nu \cdot\left(\nabla \times \mathcal{A}_{\Sigma}^{0}\left[\Phi_{s}\right]\right)\right|_{\partial \Sigma} ^{+}+\nu \cdot \nabla \times \nabla \times\left.\mathcal{A}_{\Sigma}^{0}\left[\nabla_{\partial \Sigma} \varphi_{s}\right]\right|_{\partial \Sigma} ^{+} .
\end{aligned}
$$

Define $\mathcal{M}_{\Sigma_{c}, \Sigma}^{k^{\prime}}:=\nu \times \nabla \times\left.\mathcal{A}_{\Sigma}^{k^{\prime}}\right|_{\partial \Sigma_{c}}, \mathcal{M}_{\Sigma, \Sigma_{c}}^{k^{\prime}}:=\nu \times \nabla \times\left.\mathcal{A}_{\Sigma_{c}}^{k^{\prime}}\right|_{\partial \Sigma}$, and $\mathcal{L}_{\Sigma_{c}, \Sigma}^{k^{\prime}}:=\nu \times \nabla \times \nabla \times$ $\left.\mathcal{A}_{\Sigma}^{k^{\prime}}\right|_{\partial \Sigma_{c}}, \mathcal{L}_{\Sigma, \Sigma_{c}}^{k^{\prime}}:=\nu \times \nabla \times \nabla \times\left.\mathcal{A}_{\Sigma_{c}}^{k^{\prime}}\right|_{\partial \Sigma}, k^{\prime}=0, k_{s}$. By using (2.11), (2.12) and the jump formula (2.6), there holds

$$
\left(-\frac{I}{2}+\mathcal{M}_{\Sigma_{c}}^{k_{s}}\right)\left[\Phi_{c}\right]+\mathcal{M}_{\Sigma_{c}, \Sigma}^{k_{s}}\left[\Phi_{s}\right]+\mathcal{L}_{\Sigma_{c}, \Sigma}^{k_{s}}\left[\nabla_{\partial \Sigma} \varphi_{s}\right]=\nu \times\left.\mathbf{H}_{0}\right|_{\partial \Sigma_{c}} ^{+},
$$


and

$$
\Phi_{s}+\left(\mathcal{M}_{\Sigma}^{k_{s}}-\mathcal{M}_{\Sigma}^{0}\right)\left[\Phi_{s}\right]+\left(\mathcal{M}_{\Sigma, \Sigma_{c}}^{k_{s}}-\mathcal{M}_{\Sigma, \Sigma_{c}}^{0}\right)\left[\Phi_{c}\right]+\left(\mathcal{L}_{\Sigma}^{k_{s}}-\mathcal{L}_{\Sigma}^{0}\right)\left[\nabla_{\partial \Sigma} \varphi_{s}\right]=0 .
$$

Define $\mathcal{N}_{\Sigma_{c}, \Sigma}^{k^{\prime}}:=\nu \cdot\left(\nabla \times\left.\mathcal{A}_{\Sigma}^{k^{\prime}}\right|_{\partial \Sigma_{c}}\right), \mathcal{N}_{\Sigma, \Sigma_{c}}^{k^{\prime}}:=\nu \cdot\left(\nabla \times\left.\mathcal{A}_{\Sigma_{c}}^{k^{\prime}}\right|_{\partial \Sigma}\right), k^{\prime}=0, k_{s}$. By using (2.13), noting that $\nabla \times \nabla \times=-\Delta+\nabla \nabla \cdot$, and the jump formula (2.4), one further has

$$
\begin{aligned}
& \left(\mathcal{N}_{\Sigma, \Sigma_{c}}^{k_{s}}-\mathcal{N}_{\Sigma, \Sigma_{c}}^{0}\right)\left[\Phi_{c}\right]+\left(\mathcal{N}_{\Sigma}^{k_{s}}-\mathcal{N}_{\Sigma}^{0}\right)\left[\Phi_{s}\right]+k_{s}^{2} \nu \cdot \mathcal{A}_{\Sigma}^{k_{s}}\left[\nabla_{\partial \Sigma} \varphi_{s}\right] \\
& -\nabla_{\partial \Sigma} \cdot \nabla_{\partial \Sigma} \varphi_{s}+\left(\left(\mathcal{K}_{\Sigma}^{k_{s}}\right)^{*}-\left(\mathcal{K}_{\Sigma}^{0}\right)^{*}\right)\left[\nabla_{\partial \Sigma} \cdot \nabla_{\partial \Sigma} \varphi_{s}\right]=0 .
\end{aligned}
$$

We shall need the following elementary estimates (see [1, 13]),

Lemma 2.1. There hold the following asymptotic relationships for $k_{s}$ sufficiently small:

$$
\begin{aligned}
& \mathcal{M}_{\Sigma^{\prime}, \Sigma^{\prime \prime}}^{k_{s}}=\mathcal{M}_{\Sigma^{\prime}, \Sigma^{\prime \prime}}^{0}+\mathcal{O}\left(k_{s}^{2}\right), \quad \mathcal{L}_{\Sigma^{\prime}, \Sigma^{\prime \prime}}^{k_{s}}=\mathcal{L}_{\Sigma^{\prime}, \Sigma^{\prime \prime}}^{0}+\mathcal{O}\left(k_{s}^{2}\right), \\
& \mathcal{N}_{\Sigma^{\prime}, \Sigma^{\prime \prime}}^{k_{s}}=\mathcal{N}_{\Sigma^{\prime}, \Sigma^{\prime \prime}}^{0}+\mathcal{O}\left(k_{s}^{2}\right), \quad\left(\mathcal{K}_{\Sigma_{s}^{\prime}}^{k_{s}}\right)^{*}=\left(\mathcal{K}_{\Sigma^{\prime}}^{0}\right)^{*}+\mathcal{O}\left(k_{s}^{2}\right),
\end{aligned}
$$

where $\Sigma^{\prime}, \Sigma^{\prime \prime} \in\left\{\Sigma, \Sigma_{c}, D_{1}, D_{2}, \ldots, D_{l}\right\}$. Furthermore, if $\Sigma^{\prime}=\Sigma^{\prime \prime}$ then the subscripts of the related operators in (2.17) reduces to $\Sigma^{\prime}$ only.

Based on (2.14)-(2.16) and Lemma 2.1, we can derive the following asymptotic expansion of $\mathbf{H}_{0}$, whose proof is given in Appendix A.

Lemma 2.2. Let $\mathbf{H}_{0}$ be the solution to (1.16). Then for $\omega \in \mathbb{R}_{+}$sufficiently small, there holds the following asymptotic expansion:

$$
\mathbf{H}_{0}=\nabla \times \mathcal{A}_{\Sigma_{c}}^{0}\left(-\frac{I}{2}+\mathcal{M}_{\Sigma_{c}}^{0}\right)^{-1}\left[\nu \times\left.\mathbf{H}_{0}\right|_{\partial \Sigma_{c}} ^{+}\right]+\mathcal{O}\left(k_{s}^{2}\right) \quad \text { in } \quad \mathbb{R}^{3} \backslash \overline{\Sigma_{c}} .
$$

Next we establish the asymptotic expansion of the magnetic field $\mathbf{H}$ associated with the system (1.17). First, by using standard layer potential theory, one can directly verify that $\mathbf{H}$ can be given by the following integral ansatz,

$$
\mathbf{H}=\left\{\begin{aligned}
\nabla & \times \mathcal{A}_{\Sigma_{c}}^{0}\left[\Psi_{0}\right]+\nabla \times \mathcal{A}_{\Sigma}^{0}\left[\Phi_{0}\right]+\nabla \times \nabla \times \mathcal{A}_{\Sigma}^{0}\left[\nabla_{\partial \Sigma} \varphi_{0}\right] \\
& +\nabla \times \sum_{l^{\prime}=1}^{l_{0}} \mathcal{A}_{D_{l^{\prime}}}^{0}\left[\Phi_{l^{\prime}}\right]+\nabla \times \nabla \times \sum_{l^{\prime}=1}^{l_{0}} \mathcal{A}_{D_{l^{\prime}}}^{0}\left[\nabla_{\partial D_{l^{\prime}}} \varphi_{l^{\prime}}\right] \quad \text { in } \mathbb{R}^{3} \backslash \bar{\Sigma} \\
\nabla & \times \mathcal{A}_{\Sigma_{c}}^{k_{s}}\left[\Psi_{0}\right]+\nabla \times \mathcal{A}_{\Sigma}^{k_{s}}\left[\Phi_{0}\right]+\nabla \times \nabla \times \mathcal{A}_{\Sigma}^{k_{s}}\left[\nabla_{\partial \Sigma} \varphi_{0}\right] \\
& +\nabla \times \sum_{l^{\prime}=1}^{l_{0}} \mathcal{A}_{D_{l^{\prime}}}^{k_{s}}\left[\Phi_{l^{\prime}}\right]+\nabla \times \nabla \times \sum_{l^{\prime}=1}^{l_{0}} \mathcal{A}_{D_{l^{\prime}}}^{k_{s}}\left[\nabla_{\partial D_{l^{\prime}}} \varphi_{l^{\prime}}\right] \quad \text { in } \Sigma_{s} \backslash \bigcup_{l^{\prime}=1}^{l_{0}} D_{l^{\prime}}, \\
\nabla & \times \mathcal{A}_{\Sigma_{c}}^{k_{l}}\left[\Psi_{0}\right]+\nabla \times \mathcal{A}_{\Sigma}^{k_{l}}\left[\Phi_{0}\right]+\nabla \times \nabla \times \mathcal{A}_{\Sigma}^{k_{l}}\left[\nabla_{\partial \Sigma} \varphi_{0}\right] \\
& +\nabla \times \sum_{l^{\prime}=1}^{l_{0}} \mathcal{A}_{D_{l^{\prime}}}^{k_{l}}\left[\Phi_{l^{\prime}}\right]+\nabla \times \nabla \times \sum_{l^{\prime}=1}^{l_{0}} \mathcal{A}_{D_{l^{\prime}}}^{k_{l}}\left[\nabla_{\partial D_{l^{\prime}}} \varphi_{l^{\prime}}\right] \quad \text { in } D_{l}, l=1,2, \cdots, l_{0},
\end{aligned}\right.
$$

where the densities $\left(\Psi_{0}, \Phi_{0}, \Phi_{1}, \Phi_{2}, \ldots, \Phi_{l_{0}}\right)$ satisfy $\left(\Psi_{0}, \Phi_{0}, \Phi_{1}, \Phi_{2}, \ldots, \Phi_{l_{0}}\right) \in \mathrm{TH}\left(\operatorname{div}, \partial \Sigma_{c}\right) \otimes$ $\mathrm{TH}(\operatorname{div}, \partial \Sigma) \otimes \mathrm{TH}\left(\operatorname{div}, \partial D_{1}\right) \otimes \mathrm{TH}\left(\operatorname{div}, \partial D_{2}\right) \otimes \cdots \otimes \mathrm{TH}\left(\operatorname{div}, \partial D_{l_{0}}\right)$. The densities $\left(\varphi_{0}, \varphi_{1}, \ldots, \varphi_{l_{0}}\right)$ belongs to $H^{1}(\partial \Sigma) \otimes H^{1}\left(\partial D_{1}\right) \otimes \cdots \otimes H^{1}\left(\partial D_{l_{0}}\right)$. We show the uniqueness of the densities in (B.2) when $\omega$ is sufficiently small. Based on the above integral representation, we can show the following asymptotic expansion of the magnetic field $\mathbf{H}$, where proof is given in Appendix B. 
Lemma 2.3. Let $\mathbf{H}$ be the solution to (1.17). Then for $\omega \in \mathbb{R}_{+}$sufficiently small, there holds the following asymptotic expansion:

$$
\mathbf{H}=\nabla \times \mathcal{A}_{\Sigma_{c}}^{0}\left[\Psi_{0}^{(0)}\right]+\nabla \sum_{l=1}^{l_{0}} \mathcal{S}_{D_{l}}^{0}\left[\varphi_{l}^{(0)}\right]+\mathcal{O}(\omega) \quad \text { in } \quad \mathbb{R}^{3} \backslash \overline{\Sigma_{c}},
$$

where $\left(\varphi_{1}^{(0)}, \varphi_{2}^{(0)}, \ldots, \varphi_{l_{0}}^{(0)}\right) \in L^{2}\left(\partial D_{1}\right) \otimes L^{2}\left(\partial D_{2}\right) \otimes \cdots \otimes L^{2}\left(\partial D_{l_{0}}\right)$ are solutions to the following integral equations:

$$
\begin{aligned}
& \left(\varsigma_{l} I-\left(\mathcal{K}_{D_{l}}^{0}\right)^{*}+\mathcal{P}_{D_{l}, \Sigma_{c}} \mathcal{L}_{\Sigma_{c}, D_{l}}^{0}\right)\left[\varphi_{l}^{(0)}\right]-\sum_{l^{\prime} \neq l}^{l_{0}}\left(\mathcal{K}_{D_{l}, D_{l^{\prime}}}^{0}-\mathcal{P}_{D_{l}, \Sigma_{c}} \mathcal{L}_{\Sigma_{c}, D_{l^{\prime}}}^{0}\right)\left[\varphi_{l^{\prime}}^{(0)}\right] \\
= & \mathcal{P}_{D_{l}, \Sigma_{c}}\left[\nu \times\left.\mathbf{H}^{0}\right|_{\partial \Sigma_{c}}\right] \quad \text { on } \quad \partial D_{l}, l=1,2, \ldots, l_{0},
\end{aligned}
$$

where

$$
\varsigma_{l}:=\frac{\mu_{l}+\mu_{0}}{2\left(\mu_{l}-\mu_{0}\right)},
$$

and the operators $\mathcal{K}_{D_{l}, D_{l^{\prime}}}^{0}$ are defined in (B.5) and $\mathcal{P}_{D_{l}, \Sigma_{c}}$ are defined in (B.11), l, $l^{\prime}=$ $1,2, \ldots, l_{0}$. Furthermore, $\Psi_{0}^{(0)} \in \mathrm{TH}\left(\operatorname{div}, \partial \Sigma_{c}\right)$ is the solution to the following integral equation:

$$
\Psi_{0}^{(0)}=\left(-\frac{I}{2}+\mathcal{M}_{\Sigma_{c}}^{0}\right)^{-1}\left[\nu \times\left.\mathbf{H}^{0}\right|_{\partial \Sigma_{c}}\right]-\sum_{l^{\prime}=1}^{l_{0}}\left(-\frac{I}{2}+\mathcal{M}_{\Sigma_{c}}^{0}\right)^{-1} \mathcal{L}_{\Sigma_{c}, D_{l^{\prime}}}^{0}\left[\varphi_{l^{\prime}}^{(0)}\right] .
$$

Here, we denote by $\mathbf{H}^{0}$ the leading-order term of $\mathbf{H}$ with respect to $\omega$, i.e., $\mathbf{H}=\mathbf{H}^{0}+\mathcal{O}(\omega)$.

2.3. Asymptotic expansions of the static magnetic fields with respect to the anomaly size. In this section, we make further asymptotic expansions of the static magnetic fields derived in the previous subsection with respect to the size of the magnetized anomalies. In fact, from a practical point of view, the size of the magnetized anomalies $\left(D_{l} ; \mu_{l}, \sigma_{l}\right), l=1,2, \ldots, l_{0}$, introduced in (1.11), is much smaller than the size of the Earth. Hence, we can assume that

$$
D_{l}=\delta \Omega+\mathbf{z}_{l}, \quad l=1,2, \ldots, l_{0},
$$

where $\Omega$ is a bounded Lipschitz domain in $\mathbb{R}^{3}$ with $\Omega \Subset \Sigma$, and $\delta \in \mathbb{R}_{+}$is sufficiently small. Furthermore, we assume that $D_{l}, l=1,2, \ldots, l_{0}$ are sparsely distributed and $\mathbf{z}_{l}$, $l=1,2, \ldots, l_{0}$, are sufficiently far away from $\partial \Sigma_{s}$ such that $\mathbf{x}-\mathbf{z}_{l} \gg \delta$, for any $\mathbf{x} \in \partial \Sigma_{s}$. With the above preparations, we are in a position to derive the asymptotic expansions of the static geomagnetic fields with respect to the size of the magnetized anomalies. To that end, we define a matrix operator $\mathbb{M}_{D}$ on $L^{2}\left(\partial D_{1}\right) \otimes L^{2}\left(\partial D_{2}\right) \otimes \ldots \otimes L^{2}\left(\partial D_{l_{0}}\right)$ by

$$
\mathbb{M}_{D}:=\left[\begin{array}{cccc}
\mathcal{Q}_{D_{1}} & \mathcal{Q}_{D_{1}, D_{2}} & \cdots & \mathcal{Q}_{D_{1}, D_{l_{0}}} \\
\mathcal{Q}_{D_{2}, D_{1}} & \mathcal{Q}_{D_{2}} & \cdots & \mathcal{Q}_{D_{2}, D_{l_{0}}} \\
\vdots & \vdots & \ddots & \\
\mathcal{Q}_{D_{l_{0}}, D_{1}} & \mathcal{Q}_{D_{l_{0}}, D_{2}} & \cdots & \mathcal{Q}_{D_{l_{0}}}
\end{array}\right]
$$

where $\mathcal{Q}_{D_{l}, D_{l^{\prime}}}:=\mathcal{K}_{D_{l}, D_{l^{\prime}}}^{0}-\mathcal{P}_{D_{l}, \Sigma_{c}} \mathcal{L}_{\Sigma_{c}, D_{l^{\prime}}}^{0}$ and $\mathcal{K}_{D_{l}, D_{l^{\prime}}}^{0}$ are defined in (B.5), $l, l^{\prime}=1,2, \ldots, l_{0}$.

We first have the following lemma

Lemma 2.4. Suppose $D_{l}, l=1,2, \ldots, l_{0}$ are defined in 2.24) with $\delta \in \mathbb{R}_{+}$sufficiently small. Let $\mathbb{M}_{D}$ be defined in (2.25). If $\left(\phi_{0}, \phi_{1}, \phi_{2}, \ldots, \phi_{l_{0}}\right) \in L_{0}^{2}\left(\partial D_{1}\right) \otimes L_{0}^{2}\left(\partial D_{2}\right) \otimes \ldots \otimes$ 
$L_{0}^{2}\left(\partial D_{l_{0}}\right)$, where $L_{0}^{2}\left(\partial D_{l}\right), 1 \leq l \leq l_{0}$ is the $L^{2}\left(\partial D_{l}\right)$ space whose elements have zero means on the boundary, then there holds

$$
\mathbb{M}_{D}\left(\phi_{0}, \phi_{1}, \phi_{2}, \ldots, \phi_{l_{0}}\right)^{T}=\mathbb{N}_{\Omega}\left(\tilde{\phi}_{0}, \tilde{\phi}_{1}, \tilde{\phi}_{2}, \ldots, \tilde{\phi}_{l_{0}}\right)^{T}+\mathcal{O}\left(\delta^{2}\right),
$$

where $\mathbb{N}_{\Omega}$ is an $l_{0} \times l_{0}$ matrix-valued operator defined by

$$
\mathbb{N}_{\Omega}:=\operatorname{diag}\left(\left(\mathcal{K}_{\Omega}^{0}\right)^{*},\left(\mathcal{K}_{\Omega}^{0}\right)^{*}, \ldots,\left(\mathcal{K}_{\Omega}^{0}\right)^{*}\right) .
$$

Here, the density functions $\tilde{\phi}_{l}, l=0,1, \ldots, l_{0}$, are defined as, $\tilde{\phi}_{l}(\tilde{\mathbf{y}}):=\phi_{l}(\mathbf{y})$, where $\tilde{\mathbf{y}}=$ $\delta^{-1}\left(\mathbf{y}-\mathbf{z}_{l}\right) \in \partial \Omega, l \in\left\{1,2, \ldots, l_{0}\right\}$.

Proof. For any $\mathbf{x} \in \partial D_{l}, \mathbf{y} \in \partial D_{l^{\prime}}$ and $\tilde{\mathbf{x}}=\delta^{-1}\left(\mathbf{x}-\mathbf{z}_{l}\right), \tilde{\mathbf{y}}=\delta^{-1}\left(\mathbf{y}-\mathbf{z}_{l^{\prime}}\right) \in \partial \Omega$, where $l, l^{\prime} \in\left\{1,2, \ldots, l_{0}\right\}$, one can show that

$$
\begin{aligned}
\mathcal{P}_{D_{l}, \Sigma_{c}} \mathcal{L}_{\Sigma_{c}, D_{l^{\prime}}}^{0}[\phi](\mathbf{x}) & =\nu_{\mathbf{x}} \cdot \nabla_{\mathbf{x}} \times \mathcal{A}_{\Sigma_{c}}^{0}\left(-\frac{I}{2}+\mathcal{M}_{\Sigma_{c}}^{0}\right)^{-1} \nu \times \nabla \int_{\partial D_{l^{\prime}}} \Gamma_{0}(\cdot-\mathbf{y}) \phi(\mathbf{y}) d s_{\mathbf{y}} \\
& =\delta \nu_{\tilde{\mathbf{x}}} \cdot \nabla_{\tilde{\mathbf{x}}} \times \mathcal{A}_{\Sigma_{c}}^{0}\left(-\frac{I}{2}+\mathcal{M}_{\Sigma_{c}}^{0}\right)^{-1} \nu \times \nabla \int_{\partial \Omega} \Gamma_{0}\left(\cdot-\delta \tilde{\mathbf{y}}-\mathbf{z}_{l}\right) \tilde{\phi}(\tilde{\mathbf{y}}) d s_{\tilde{\mathbf{y}}} \\
& =\delta \nu_{\tilde{\mathbf{x}}} \cdot \nabla_{\tilde{\mathbf{x}}} \times \mathcal{A}_{\Sigma_{c}}^{0}\left(-\frac{I}{2}+\mathcal{M}_{\Sigma_{c}}^{0}\right)^{-1} \nu \times \nabla \Gamma_{0}\left(\cdot-\mathbf{z}_{l}\right) \int_{\partial \Omega} \tilde{\phi}_{l^{\prime}}(\tilde{\mathbf{y}}) d s_{\tilde{\mathbf{y}}}+\mathcal{O}\left(\delta^{2}\right) \\
& =\mathcal{O}\left(\delta^{2}\right) .
\end{aligned}
$$

Similarly one can show that

$$
\mathcal{K}_{D_{l}, D_{l^{\prime}}}^{0}[\phi]=\mathcal{O}\left(\delta^{2}\right), \quad l \neq l^{\prime}, \quad \text { and } \quad\left(\mathcal{K}_{D_{l^{\prime}}}^{0}\right)^{*}[\phi]=\left(\mathcal{K}_{\Omega}^{0}\right)^{*}[\tilde{\phi}]+\mathcal{O}\left(\delta^{2}\right) .
$$

By substituting (2.28) and (2.29) back into (2.25), one readily has (2.26).

The proof is complete.

In what follows, we let $\mathbf{H}_{0}^{0}$ and $\mathbf{H}^{0}$ respectively be the leading-order terms of $\mathbf{H}_{0}$ and $\mathbf{H}$ with respect to the low-frequency $\omega$; see (2.18) and (2.20). By the asymptotic estimate in Lemma 2.4, one can derive the following asymptotic property of the densities $\varphi_{1}^{(0)}, \varphi_{2}^{(0)}, \ldots, \varphi_{l_{0}}^{(0)}$ and $\Psi_{0}^{(0)}$ defined in Lemma 2.3 ,

Lemma 2.5. Let $D_{l}, l=1,2, \ldots, l_{0}$ be given by 2.24). Let $\varphi_{1}^{(0)}, \varphi_{2}^{(0)}, \ldots, \varphi_{l_{0}}^{(0)}$ and $\Psi_{0}^{(0)}$ be defined in Lemma 2.3 and $\tilde{\varphi}_{l}^{(0)}(\tilde{\mathbf{y}}):=\varphi_{l}^{(0)}(\mathbf{y})$, where $\tilde{\mathbf{y}}=\delta^{-1}\left(\mathbf{y}-\mathbf{z}_{l}\right) \in \partial \Omega, l=1,2, \ldots, l_{0}$. Then there hold the following estimates:

$$
\tilde{\varphi}_{l}^{(0)}=\left(\varsigma_{l} I-\left(\mathcal{K}_{\Omega}^{0}\right)^{*}\right)^{-1}[\nu] \cdot \mathbb{H}\left(\mathbf{z}_{l}\right)+\mathcal{O}(\delta), \quad l=1,2, \ldots, l_{0},
$$

and

$$
\begin{aligned}
\Psi_{0}^{(0)}= & \left(-\frac{I}{2}+\mathcal{M}_{\Sigma_{c}}^{0}\right)^{-1}\left[\nu \times\left.\mathbf{H}^{0}\right|_{\partial \Sigma_{c}}\right]+\mathcal{O}\left(\delta^{4}\right)+ \\
& \delta^{3}\left(-\frac{I}{2}+\mathcal{M}_{\Sigma_{c}}^{0}\right)^{-1}\left[\nu \times \sum_{l^{\prime}=1}^{l_{0}} \nabla^{2} \Gamma_{0}\left(\cdot-\mathbf{z}_{l}\right) \int_{\partial \Omega} \tilde{\mathbf{y}}\left(\varsigma_{l} I-\left(\mathcal{K}_{\Omega}^{0}\right)^{*}\right)^{-1}[\nu](\tilde{\mathbf{y}}) d s_{\tilde{\mathbf{y}}} \mathbb{H}\left(\mathbf{z}_{l}\right)\right],
\end{aligned}
$$

where $\varsigma_{l}$ is defined by (2.22) and $\mathbb{H}$ is defined by

$$
\mathbb{H}:=\nabla \times \mathcal{A}_{\Sigma_{c}}^{0}\left(-\frac{I}{2}+\mathcal{M}_{\Sigma_{c}}^{0}\right)^{-1}\left[\nu \times\left.\mathbf{H}^{0}\right|_{\partial \Sigma_{c}}\right]
$$


Proof. First, by using (2.25) one can rewrite (2.21) in the following form,

$$
\left(\varsigma-\mathbb{M}_{D}\right)[\varphi]=\mathbf{H}_{c},
$$

where $\varsigma:=\operatorname{diag}\left(\varsigma_{1}, \varsigma_{2}, \ldots, \varsigma_{l_{0}}\right)$ and

$$
\mathbf{H}_{c}:=\left(\mathcal{P}_{D_{1}, \Sigma_{c}}\left[\nu \times\left.\mathbf{H}^{0}\right|_{\partial \Sigma_{c}}\right], \mathcal{P}_{D_{2}, \Sigma_{c}}\left[\nu \times\left.\mathbf{H}^{0}\right|_{\partial \Sigma_{c}}\right], \ldots, \mathcal{P}_{D_{l_{0}}, \Sigma_{c}}\left[\nu \times\left.\mathbf{H}^{0}\right|_{\partial \Sigma_{c}}\right]\right)^{T} .
$$

We shall analyze the right hand side term in (2.33). By definition,

$$
\mathcal{P}_{D_{l}, \Sigma_{c}}\left[\nu \times\left.\mathbf{H}^{0}\right|_{\partial \Sigma_{c}}\right]=\nu \cdot \nabla \times \mathcal{A}_{\Sigma_{c}}^{0}\left(-\frac{I}{2}+\mathcal{M}_{\Sigma_{c}}^{0}\right)^{-1}\left[\nu \times\left.\mathbf{H}^{0}\right|_{\partial \Sigma_{c}}\right] \quad \text { on } \partial D_{l} .
$$

which together with the Taylor series expansion of $\mathbb{H}$ on $\mathbf{z}_{l}$, i.e.,

$$
\mathbb{H}(\mathbf{y})=\mathbb{H}\left(\mathbf{z}_{l}\right)+\nabla \mathbb{H}\left(\mathbf{z}_{l}\right)\left(\mathbf{y}-\mathbf{z}_{l}\right)+\mathcal{O}\left(\left\|\mathbf{y}-\mathbf{z}_{l}\right\|^{2}\right), \quad \mathbf{y} \in \partial D_{l},
$$

readily yields that

$$
\mathcal{P}_{D_{l}, \Sigma_{c}}\left[\nu \times\left.\mathbf{H}^{0}\right|_{\partial \Sigma_{c}}\right]=\nu \cdot \mathbb{H}\left(\mathbf{z}_{l}\right)+\mathcal{O}(\delta) \quad \text { on } \quad \partial \Omega .
$$

By combing (2.26), (2.27), (2.33) and (2.36), one thus has (2.30). Next, by taking integration on both sides of (2.21) over $\partial D_{l}$ and using integration by parts, one can derive that

$$
\int_{\partial D_{l}}\left(\varsigma_{l} I-\left(\mathcal{K}_{D_{l}}^{0}\right)^{*}+\mathcal{P}_{D_{l}, \Sigma_{c}} \mathcal{L}_{\Sigma_{c}, D_{l}}^{0}\right)\left[\varphi_{l}^{(0)}\right] d s=\left(\varsigma_{l}-\frac{1}{2}\right) \int_{\partial D_{l}} \varphi_{l}^{(0)} d s=0
$$

where the well-known result $\mathcal{K}_{D_{l}}^{0}[1]=1 / 2$ is used (see, e.g., [2]). Note that $\left(\varsigma_{l}-\frac{1}{2}\right) \neq 0$, one thus has

$$
\int_{\partial D_{l}} \varphi_{l}^{(0)} d s=0, \quad l=1,2, \ldots, l_{0} .
$$

By using (2.28), one can further show that for $\mathbf{x} \in \partial \Sigma_{c}$,

$$
\begin{aligned}
\mathcal{L}_{\Sigma_{c}, D_{l^{\prime}}}^{0}\left[\varphi_{l^{\prime}}^{(0)}\right](\mathbf{x})= & \nu \times \nabla \int_{\partial D_{l^{\prime}}} \Gamma_{0}(\mathbf{x}-\mathbf{y}) \varphi_{l^{\prime}}^{(0)}(\mathbf{y}) d s_{\mathbf{y}} \\
& =\delta^{2} \nu \times \nabla \int_{\partial \Omega} \Gamma_{0}\left(\mathbf{x}-\delta \tilde{\mathbf{y}}-\mathbf{z}_{l}\right) \tilde{\varphi}_{l^{\prime}}^{(0)}(\tilde{\mathbf{y}}) d s_{\tilde{\mathbf{y}}} \\
& =-\delta^{3} \nu \times \nabla^{2} \Gamma_{0}\left(\mathbf{x}-\mathbf{z}_{l}\right) \int_{\partial \Omega} \tilde{\mathbf{y}} \tilde{\varphi}_{l^{\prime}}^{(0)}(\tilde{\mathbf{y}}) d s_{\tilde{\mathbf{y}}}+\mathcal{O}\left(\delta^{4}\right) .
\end{aligned}
$$

Thus from (2.23) one immediately proves (2.31), which completes the proof.

We are in a position to present our first main result.

Theorem 2.1. Suppose $\mathbf{H}_{0}$ and $\mathbf{H}$ are solutions to (1.16) and (1.17), respectively. Suppose $D_{l}, l=1,2, \ldots, l_{0}$ satisfy (2.24), and let $\mathbf{H}_{0}^{0}$ and $\mathbf{H}^{0}$ be the leading-order terms with respect to $\omega \ll 1$ of $\mathbf{H}_{0}$ and $\mathbf{H}$, respectively. Then for $\mathbf{x} \in \mathbb{R}^{3} \backslash \bar{\Sigma}$, there holds the following asymptotic expansion:

$$
\begin{aligned}
\left(\mathbf{H}^{0}-\mathbf{H}_{0}^{0}\right)(\mathbf{x})= & \nabla \times \mathcal{A}_{\Sigma_{c}}^{0}\left(-\frac{I}{2}+\mathcal{M}_{\Sigma_{c}}^{0}\right)^{-1}\left[\nu \times\left.\left(\mathbf{H}^{0}-\mathbf{H}_{0}^{0}\right)\right|_{\partial \Sigma_{c}} ^{+}\right](\mathbf{x}) \\
& +\delta^{3} \nabla \mathcal{S}_{\Sigma_{c}}^{0}\left(-\frac{I}{2}+\left(\mathcal{K}_{\Sigma_{c}}^{0}\right)^{*}\right)^{-1}\left[\nu \cdot \nabla^{2} \sum_{l=1}^{l_{0}} \Gamma_{0}\left(\cdot-\mathbf{z}_{l}\right) \mathbf{M}_{l} \mathbb{H}\left(\mathbf{z}_{l}\right)\right](\mathbf{x}) \\
& -\delta^{3} \sum_{l=1}^{l_{0}} \nabla^{2} \Gamma_{0}\left(\mathbf{x}-\mathbf{z}_{l}\right) \mathbf{M}_{l} \mathbb{H}\left(\mathbf{z}_{l}\right)+\mathcal{O}\left(\delta^{4}\right)
\end{aligned}
$$


where $\mathbf{M}_{l}, l=1,2, \ldots, l_{0}$, are the polarization tensors defined by

$$
\mathbf{M}_{l}:=\int_{\partial \Omega} \mathbf{y}\left(\varsigma_{l} I-\left(\mathcal{K}_{\Omega}^{0}\right)^{*}\right)^{-1}[\nu](\mathbf{y}) d s_{\mathbf{y}},
$$

and $\varsigma_{l}$ and $\mathbb{H}$ are respectively defined in (2.22) and (2.32).

Before presenting the proof of Theorem 2.1, we remark that in the formula (2.40), the asymptotic expansion of $\mathbf{H}^{0}-\mathbf{H}_{0}^{0}$ contains the term $\mathbf{H}^{0}-\mathbf{H}_{0}^{0}$ on $\partial \Sigma_{c}$ (in the first term of the right hand side of (2.401) $)$, which is not directly given. However, according to our earlier discussion about the data continuation, one actually can continue the measurement data on $\Gamma$ to $\Sigma_{c}$. Nevertheless, in the next section, we shall perform further asymptotic analysis to show that this data continuation step can actually be relaxed.

Proof of Theorem 2.1. By using Taylor's series expansion, (2.30) and (2.38), one can show that

$$
\begin{aligned}
\nabla \sum_{l^{\prime}=1}^{l_{0}} \mathcal{S}_{D_{l^{\prime}}}^{0}\left[\varphi_{l^{\prime}}^{(0)}\right](\mathbf{x}) & =\sum_{l^{\prime}=1}^{l_{0}} \sum_{|\alpha|=0}^{\infty} \frac{(-1)^{|\alpha|}}{\alpha !} \nabla \int_{\partial D_{l}^{\prime}} \partial^{\alpha} \Gamma_{0}\left(\mathbf{x}-\mathbf{z}_{l}\right)\left(\mathbf{y}-\mathbf{z}_{l}\right)^{\alpha} \varphi_{l^{\prime}}^{(0)}(\mathbf{y}) d s_{\mathbf{y}} \\
& =-\delta^{3} \sum_{l^{\prime}=1}^{l_{0}} \nabla^{2} \Gamma_{0}\left(\mathbf{x}-\mathbf{z}_{l}\right) \int_{\partial \Omega} \tilde{\mathbf{y}} \tilde{\varphi}_{l^{\prime}}^{(0)}(\tilde{\mathbf{y}}) d s_{\tilde{\mathbf{y}}}+\mathcal{O}\left(\delta^{4}\right) \\
& =-\delta^{3} \sum_{l^{\prime}=1}^{l_{0}} \nabla^{2} \Gamma_{0}\left(\mathbf{x}-\mathbf{z}_{l}\right) \int_{\partial \Omega} \tilde{\mathbf{y}}\left(\varsigma_{l} I-\left(\mathcal{K}_{\Omega}^{0}\right)^{*}\right)^{-1}[\nu](\tilde{\mathbf{y}}) d s_{\tilde{\mathbf{y}}} \mathbb{H}\left(\mathbf{z}_{l}\right)+\mathcal{O}\left(\delta^{4}\right) .
\end{aligned}
$$

By using Lemma 3.6 in [13], there holds

$$
\begin{aligned}
& \nabla \times \mathcal{A}_{\Sigma_{c}}^{0}\left(-\frac{I}{2}+\mathcal{M}_{\Sigma_{c}}^{0}\right)^{-1}\left[\nu \times \nabla^{2} \sum_{l=1}^{l_{0}} \Gamma_{0}\left(\cdot-\mathbf{z}_{l}\right) \mathbf{M}_{l} \mathbb{H}\left(\mathbf{z}_{l}\right)\right](\mathbf{x}) \\
= & \nabla \mathcal{S}_{\Sigma_{c}}^{0}\left(-\frac{I}{2}+\left(\mathcal{K}_{\Sigma_{c}}^{0}\right)^{*}\right)^{-1}\left[\nu \cdot \nabla^{2} \sum_{l=1}^{l_{0}} \Gamma_{0}\left(\cdot-\mathbf{z}_{l}\right) \mathbf{M}_{l} \mathbb{H}\left(\mathbf{z}_{l}\right)\right](\mathbf{x}), \quad \mathbf{x} \in \mathbb{R}^{3} \backslash \overline{\Sigma_{c}} .
\end{aligned}
$$

Finally, by combing (2.18), (2.20) and using (2.31), (2.42), (2.43) one can derive (3.4), which completes the proof.

\section{Further ASYmptotic ANALYSIS}

In this section, we conduct further asymptotic analysis on the leading-order term in (2.40). As remarked earlier, in (3.4), one needs to derive the magnetic field on $\partial \Sigma_{c}$ through the continuation of the measurement data on $\Gamma$. It is well-known that the data continuation is severely ill-conditioned. We next show that this data continuation can actually be relaxed. The result in this section may find important application in the practical realization of our theoretical findings. Before that, we first present an important axillary result.

Lemma 3.1 (Lemma 3.9 in [13]). Let $\mathbf{z} \in \mathbb{R}^{3}$ be fixed. Let $\mathbf{x} \in \partial B_{R}$ and suppose $\|\mathbf{z}\|<R$. There holds the following asymptotic expansion

$$
\nabla \Gamma_{0}(\mathbf{x}-\mathbf{z})=\sum_{n=0}^{\infty} \sum_{m=-n}^{n} \frac{(n+1) Y_{n}^{m}(\hat{\mathbf{x}}) \hat{\mathbf{x}}-\nabla_{S} Y_{n}^{m}(\hat{\mathbf{x}})}{(2 n+1) R^{n+2}} \overline{Y_{n}^{m}(\hat{\mathbf{z}})}\|\mathbf{z}\|^{n}
$$

where $\hat{\mathbf{z}}=\mathbf{z} /\|\mathbf{z}\|$ and $\hat{\mathbf{x}}=\mathbf{x} /\|\mathbf{x}\| . Y_{n}^{m}$ is the spherical harmonics of order $m$ and degree $n$. 
In the sequel, we define

$$
\mathbf{N}_{n+1}^{m}(\hat{\mathbf{x}})=(n+1) Y_{n}^{m}(\hat{\mathbf{x}}) \hat{\mathbf{x}}-\nabla_{S} Y_{n}^{m}(\hat{\mathbf{x}}),
$$

for $n \in \mathbb{N} \cup\{0\}$ and $m=-n,-n+1, \ldots, n-1, n$, and

$$
\mathbf{Q}_{n-1}^{m}(\hat{\mathbf{x}}):=\nabla_{S} Y_{n}^{m}(\hat{\mathbf{x}})+n Y_{n}^{m}(\hat{\mathbf{x}}) \hat{\mathbf{x}}, \quad \mathbf{T}_{n}^{m}(\hat{\mathbf{x}}):=\nabla_{S} Y_{n}^{m}(\hat{\mathbf{x}}) \times \hat{\mathbf{x}}
$$

for $n \in \mathbb{N}$ and $m=-n,-n+1, \ldots, n-1, n$. Note that $\mathbf{N}_{n+1}^{m}, \mathbf{Q}_{n-1}^{m}(\hat{\mathbf{x}})$ and $\mathbf{T}_{n}^{m}(\hat{\mathbf{x}})$ are spherical harmonics of order $n$. Rewrite the formula (2.40) as

$$
\left(\mathbf{H}^{0}-\mathbf{H}_{0}^{0}\right)(\mathbf{x})=\tilde{\mathbf{H}}_{1}(\mathbf{x})+\delta^{3} \tilde{\mathbf{H}}_{2}(\mathbf{x})-\delta^{3} \tilde{\mathbf{H}}_{3}(\mathbf{x})+\mathcal{O}\left(\delta^{4}\right),
$$

where

$$
\widetilde{\mathbf{H}}_{1}(\mathbf{x}):=\nabla \times \mathcal{A}_{\Sigma_{c}}^{0}\left(-\frac{I}{2}+\mathcal{M}_{\Sigma_{c}}^{0}\right)^{-1}\left[\nu \times\left.\left(\mathbf{H}^{0}-\mathbf{H}_{0}^{0}\right)\right|_{\partial \Sigma_{c}} ^{+}\right](\mathbf{x}),
$$

and

$$
\widetilde{\mathbf{H}}_{2}(\mathbf{x}):=\nabla \mathcal{S}_{\Sigma_{c}}^{0}\left(-\frac{I}{2}+\left(\mathcal{K}_{\Sigma_{c}}^{0}\right)^{*}\right)^{-1}\left[\nu \cdot \nabla^{2} \sum_{l=1}^{l_{0}} \Gamma_{0}\left(\cdot-\mathbf{z}_{l}\right) \mathbf{M}_{l} \mathbb{H}\left(\mathbf{z}_{l}\right)\right](\mathbf{x})
$$

and

$$
\widetilde{\mathbf{H}}_{3}(\mathbf{x}):=\sum_{l=1}^{l_{0}} \nabla^{2} \Gamma_{0}\left(\mathbf{x}-\mathbf{z}_{l}\right) \mathbf{M}_{l} \mathbb{H}\left(\mathbf{z}_{l}\right) .
$$

We next analyze the above terms one by one. First, by using Lemma 3.1. one can show that

$$
\begin{aligned}
\widetilde{\mathbf{H}}_{1}(\mathbf{x})= & \int_{\partial \Sigma_{c}} \nabla_{\mathbf{x}} \Gamma_{0}(\mathbf{x}-\mathbf{y}) \times\left(-\frac{I}{2}+\mathcal{M}_{\Sigma_{c}}^{0}\right)^{-1}\left[\nu \times\left.\left(\mathbf{H}^{0}-\mathbf{H}_{0}^{0}\right)\right|_{\partial \Sigma_{c}} ^{+}\right](\mathbf{y}) d s_{\mathbf{y}} \\
= & \sum_{n=0}^{\infty} \sum_{m=-n}^{n} \frac{1}{(2 n+1)\|\mathbf{x}\|^{n+2}} \mathbf{N}_{n+1}^{m}(\hat{\mathbf{x}}) \times \\
& \int_{\partial \Sigma_{c}} \overline{Y_{n}^{m}(\hat{\mathbf{y}})}\|\mathbf{y}\|^{n}\left(-\frac{I}{2}+\mathcal{M}_{\Sigma_{c}}^{0}\right)^{-1}\left[\nu \times\left.\left(\mathbf{H}^{0}-\mathbf{H}_{0}^{0}\right)\right|_{\partial \Sigma_{c}} ^{+}\right](\mathbf{y}) d s_{\mathbf{y}} \\
= & \frac{1}{3}\|\mathbf{x}\|^{-3} \sum_{m=-1}^{1} \mathbf{N}_{2}^{m}(\hat{\mathbf{x}}) \times \int_{\partial \Sigma_{c}} \overline{Y_{1}^{m}(\hat{\mathbf{y}})}\|\mathbf{y}\| \widetilde{\mathbf{H}}_{1}^{1}(\mathbf{y}) d s_{\mathbf{y}}+\mathcal{O}\left(\|\mathbf{x}\|^{-4}\right),
\end{aligned}
$$

where

$$
\widetilde{\mathbf{H}}_{1}^{1}(\mathbf{y}):=\left(-\frac{I}{2}+\mathcal{M}_{\Sigma_{c}}^{0}\right)^{-1}\left[\nu \times\left.\left(\mathbf{H}^{0}-\mathbf{H}_{0}^{0}\right)\right|_{\partial \Sigma_{c}} ^{+}\right](\mathbf{y}) .
$$

Similarly, one can show that

$$
\begin{aligned}
\widetilde{\mathbf{H}}_{2}(\mathbf{x})= & \int_{\partial \Sigma_{c}} \nabla_{\mathbf{x}} \Gamma_{0}(\mathbf{x}-\mathbf{y})\left(-\frac{I}{2}+\left(\mathcal{K}_{\Sigma_{c}}^{0}\right)^{*}\right)^{-1}\left[\nu \cdot \nabla^{2} \sum_{l=1}^{l_{0}} \Gamma_{0}\left(\cdot-\mathbf{z}_{l}\right) \mathbf{M}_{l} \mathbb{H}\left(\mathbf{z}_{l}\right)\right](\mathbf{y}) d s_{\mathbf{y}} \\
= & \sum_{n=0}^{\infty} \sum_{m=-n}^{n} \frac{1}{(2 n+1)\|\mathbf{x}\|^{n+2}} \mathbf{N}_{n+1}^{m}(\hat{\mathbf{x}}) \\
& \left(-\frac{I}{2}+\left(\mathcal{K}_{\Sigma_{c}}^{0}\right)^{*}\right)^{-1}\left[\nu \cdot \nabla^{2} \sum_{l=1}^{l_{0}} \Gamma_{0}\left(\cdot-\mathbf{z}_{l}\right) \mathbf{M}_{l} \mathbb{H}\left(\mathbf{z}_{l}\right)\right](\mathbf{y}) d s_{\mathbf{y}} \\
= & \frac{1}{3}\|\mathbf{x}\|^{-3} \sum_{m=-1}^{1} \mathbf{N}_{2}^{m}(\hat{\mathbf{x}}) \int_{\partial \Sigma_{c}} \frac{Y_{1}^{m}(\hat{\mathbf{y}})}{}\|\mathbf{y}\| \widetilde{H}_{2}^{1}(\mathbf{y}) d s_{\mathbf{y}}+\mathcal{O}\left(\|\mathbf{x}\|^{-4}\right),
\end{aligned}
$$


where

$$
\widetilde{H}_{2}^{1}(\mathbf{y}):=\left(-\frac{I}{2}+\left(\mathcal{K}_{\Sigma_{c}}^{0}\right)^{*}\right)^{-1}\left[\nu \cdot \nabla^{2} \sum_{l=1}^{l_{0}} \Gamma_{0}\left(\cdot-\mathbf{z}_{l}\right) \mathbf{M}_{l} \mathbb{H}\left(\mathbf{z}_{l}\right)\right](\mathbf{y}) .
$$

To analyze $\widetilde{\mathbf{H}}_{3}(\mathbf{x})$, we first note that by differentiating both sides of (3.1), there holds

$$
\nabla^{2} \Gamma_{0}(\mathbf{x}-\mathbf{z})=\sum_{n=0}^{\infty} \sum_{m=-n}^{n} \frac{\mathbf{A}_{n}^{m}(\hat{\mathbf{x}})}{(2 n+1)\|\mathbf{x}\|^{n+3}} \overline{Y_{n}^{m}(\hat{\mathbf{z}})}\|\mathbf{z}\|^{n},
$$

where

$$
\mathbf{A}_{n}^{m}(\hat{\mathbf{x}}):=(n+1)\left(\hat{\mathbf{x}} \nabla_{S} Y_{n}^{m}(\hat{\mathbf{x}})^{T}+Y_{n}^{m}(\hat{\mathbf{x}})\left(I-\hat{\mathbf{x}} \hat{\mathbf{x}}^{T}\right)\right)-\nabla_{S}^{2} Y_{n}^{m}(\hat{\mathbf{x}})-(n+2) \mathbf{N}_{n+1}^{m}(\hat{\mathbf{x}}) \hat{\mathbf{x}}^{T},
$$

and especially when $n=0$,

$$
\mathbf{A}_{0}^{0}(\hat{\mathbf{x}})=Y_{0}^{0}(\hat{\mathbf{x}})\left(I-3 \hat{\mathbf{x}} \hat{\mathbf{x}}^{T}\right)=\frac{1}{2} \sqrt{\frac{1}{\pi}}\left(I-3 \hat{\mathbf{x}} \hat{\mathbf{x}}^{T}\right) .
$$

Thus one can derive that

$$
\begin{aligned}
\widetilde{\mathbf{H}}_{3}(\mathbf{x}) & =\sum_{l=1}^{l_{0}} \sum_{n=0}^{\infty} \sum_{m=-n}^{n} \frac{\overline{Y_{n}^{m}\left(\hat{\mathbf{z}}_{l}\right)}\left\|\mathbf{z}_{\mathbf{l}}\right\|^{n}}{(2 n+1)\|\mathbf{x}\|^{n+3}} \mathbf{A}_{n}^{m}(\hat{\mathbf{x}}) \mathbf{M}_{l} \mathbb{H}\left(\mathbf{z}_{l}\right) \\
& =\frac{1}{4 \pi\|\mathbf{x}\|^{3}} \sum_{l=1}^{l_{0}}\left(I-3 \hat{\mathbf{x}} \hat{\mathbf{x}}^{T}\right) \mathbf{M}_{l} \mathbb{H}\left(\mathbf{z}_{l}\right)+\mathcal{O}\left(\|\mathbf{x}\|^{-4}\right) .
\end{aligned}
$$

Using the above results, we can further establish the following lemma.

Lemma 3.2. Let $\mathbb{H}$ and $\mathbf{M}_{l}$ be defined in (2.32) and 2.41, respectively. Then there holds

$$
\frac{\delta^{3}}{\|\mathbf{x}\|^{3}}\left(\overline{\mathbf{D}}_{0} \sum_{l=1}^{l_{0}} \mathbf{M}_{l} \mathbb{H}\left(\mathbf{z}_{l}\right)+\mathcal{O}\left(\|\mathbf{x}\|^{-2}\right)\right)=\int_{\mathbb{S}} \overline{\mathbf{Q}_{0}(\hat{\mathbf{x}})}\left(\mathbf{H}^{0}-\mathbf{H}_{0}^{0}\right)(\mathbf{x}) d s+\mathcal{O}\left(\delta^{4}\right),
$$

where $\mathbf{D}_{0}$ and $\mathbf{Q}_{0}(\hat{\mathbf{x}})$ is a 3-by-3 matrix given by

$$
\mathbf{D}_{0}:=-\frac{1}{6}\left(\mathbf{a}_{0,1}^{0,-1}, \mathbf{a}_{0,1}^{0,0}, \mathbf{a}_{0,1}^{0,1}\right)^{T}
$$

and

$$
\mathbf{Q}_{0}(\hat{\mathbf{x}}):=\left(\mathbf{Q}_{0}^{-1}(\hat{\mathbf{x}}), \mathbf{Q}_{0}^{0}(\hat{\mathbf{x}}), \mathbf{Q}_{0}^{1}(\hat{\mathbf{x}})\right)^{T}
$$

respectively. $\mathbf{a}_{n^{\prime}, n}^{m^{\prime}, m}$ is defined in (C.9).

Proof. It can be seen that $\mathbf{Q}_{n-1}^{m}(\hat{\mathbf{x}})$ and $\mathbf{N}_{n+1}^{m}(\hat{\mathbf{x}})$ are orthogonal to each other in $L^{2}(\mathbb{S})$ (see [12,20]), where $\mathbb{S}$ stands for the unit sphere, that is

$$
\int_{\mathbb{S}} \mathbf{N}_{n+1}^{m}(\hat{\mathbf{x}}) \cdot \overline{\mathbf{Q}_{n^{\prime}}^{m^{\prime}}(\hat{\mathbf{x}})} d s=0,
$$

for any $n, n^{\prime} \in \mathbb{N} \cup\{0\}, m=-n,-n+1, \ldots, n-1, n$ and $m^{\prime}=-n^{\prime},-n^{\prime}+1, \ldots, n^{\prime}-1, n^{\prime}$. We show that $\mathbf{Q}_{0}^{m^{\prime}}(\hat{\mathbf{x}}), m^{\prime}=-1,0,1$, are orthogonal to $\mathbf{A}_{n}^{m}(\hat{\mathbf{x}}) \xi$ for any $\xi \in \mathbb{R}^{3}$ and $n \neq$ 0,2 , where $\mathbf{A}_{n}^{m}(\hat{\mathbf{x}})$ is defined in (3.10). In fact, by using (C.13) in Appendix C and the orthogonality of the vectorial spherical harmonics, one immediately obtains

$$
\int_{\mathbb{S}} \overline{\mathbf{Q}_{0}^{m^{\prime}}(\hat{\mathbf{x}})} \cdot\left(\mathbf{A}_{n}^{m}(\hat{\mathbf{x}}) \xi\right) d s=0 \quad \text { for any } n \neq 0,2
$$


Note that if $n=2$, then by (C.13), one has

$$
\begin{aligned}
\mathbf{A}_{2}^{m}(\hat{\mathbf{x}}) \xi= & \sum_{m^{\prime}=m-1}^{m+1}\left(\left(\mathbf{c}_{1,2}^{m^{\prime}, m}\right)^{T} \xi \mathbf{N}_{2}^{m^{\prime}}(\hat{\mathbf{x}})+\left(\mathbf{c}_{3,2}^{m^{\prime}, m}\right)^{T} \xi \mathbf{N}_{4}^{m^{\prime}}(\hat{\mathbf{x}})\right. \\
& \left.+\left(\mathbf{d}_{1,2}^{m^{\prime}, m}\right)^{T} \xi \mathbf{Q}_{0}^{m^{\prime}}(\hat{\mathbf{x}})+\left(\mathbf{d}_{3,2}^{m^{\prime}, m}\right)^{T} \xi \mathbf{Q}_{2}^{m^{\prime}}(\hat{\mathbf{x}})\right),
\end{aligned}
$$

where $\mathbf{c}_{n^{\prime}, n}^{m^{\prime}, m}$ and $\mathbf{d}_{n^{\prime}, n}^{m^{\prime}, m}$ are defined in (C.14) and (C.15), respectively. Thus

$$
\int_{\mathbb{S}} \overline{\mathbf{Q}_{0}^{m^{\prime}}(\hat{\mathbf{x}})} \cdot\left(\mathbf{A}_{2}^{m}(\hat{\mathbf{x}}) \xi\right) d s=\frac{1}{3}\left(\mathbf{d}_{1,2}^{m^{\prime}, m}\right)^{T} \xi
$$

By using the orthogonality of $\mathbf{N}_{3}^{m}(\hat{\mathbf{x}})$ and $Y_{1}^{m^{\prime}}, m, m^{\prime} \in\{-1,0,1\}$, one has

$$
\int_{\mathbb{S}} \overline{Y_{1}^{m^{\prime}}(\hat{\mathbf{x}})}\left(3 Y_{2}^{m}(\hat{\mathbf{x}}) \hat{\mathbf{x}}-\nabla_{S} Y_{2}^{m}(\hat{\mathbf{x}})\right) d s=0
$$

which indicates $\mathbf{a}_{1,2}^{m^{\prime}, m}=3 \mathbf{b}_{1,2}^{m^{\prime}, m}$ by using (C.9). Together with (3.20) and (C.15), one thus has

$$
\int_{\mathbb{S}} \overline{\mathbf{Q}_{0}^{m^{\prime}}(\hat{\mathbf{x}})} \cdot\left(\mathbf{A}_{2}^{m}(\hat{\mathbf{x}}) \xi\right) d s=-\frac{1}{9}\left(\mathbf{a}_{1,2}^{m^{\prime}, m}+3 \overline{\mathbf{a}_{2,1}^{m, m^{\prime}}}\right)^{T} \xi .
$$

Similarly, for $n=0$, one obtains that

$$
\int_{\mathbb{S}} \overline{\mathbf{Q}_{0}^{m^{\prime}}(\hat{\mathbf{x}})} \cdot\left(\mathbf{A}_{0}^{0}(\hat{\mathbf{x}}) \xi\right) d s=-\frac{1}{6}\left(\overline{\mathbf{a}_{0,1}^{0, m^{\prime}}}\right)^{T} \xi .
$$

Finally, by taking inner product of (3.4) and $\mathbf{Q}_{0}^{m^{\prime}}(\hat{\mathbf{x}})$ in $L^{2}(\mathbb{S})$ and by using (3.17), (3.21) and (3.22), one has

$$
\begin{aligned}
& \int_{\mathbb{S}} \overline{\mathbf{Q}_{0}^{m^{\prime}}(\hat{\mathbf{x}})} \cdot\left(\mathbf{H}^{0}-\mathbf{H}_{0}^{0}\right)(\mathbf{x}) d s \\
= & \left.-\frac{1}{6} \frac{\delta^{3}}{\|\mathbf{x}\|^{3}} \overline{\left(\mathbf{a}_{0,1}^{0, m^{\prime}}\right.}\right)^{T} \sum_{l=1}^{l_{0}} \mathbf{M}_{l} \mathbb{H}\left(\mathbf{z}_{l}\right) \\
& -\frac{1}{45} \sum_{m=-2}^{2} \frac{\delta^{3}}{\|\mathbf{x}\|^{5}}\left(\mathbf{a}_{1,2}^{m^{\prime}, m}+3 \overline{\mathbf{a}_{2,1}^{m, m^{\prime}}}\right)^{T} \sum_{l=1}^{l_{0}} \overline{Y_{2}^{m}\left(\hat{\mathbf{z}}_{l}\right)}\left\|\mathbf{z}_{l}\right\|^{2} \mathbf{M}_{l} \mathbb{H}\left(\mathbf{z}_{l}\right)+\mathcal{O}\left(\delta^{4}\right) .
\end{aligned}
$$

By rearranging the terms in equation (3.23), we finally arrive at (3.13).

The proof is complete.

Before proceeding further, we remark that the coefficient of higher order $\mathcal{O}\left(\|\mathbf{x}\|^{-2}\right)$ in (3.13) is accurately given in (3.23). One can readily see that if $\left\|\mathbf{z}_{l}\right\| /\|\mathbf{x}\|$ is small enough, that is, the radius of the measurement surface is much bigger than the length of the position vector of the magnetized anomaly, then the unknown term $\mathbf{M}_{l} \mathbb{H}\left(\mathbf{z}_{l}\right)$ is easy to recover. Thus the positions of the magnetized anomalies are easy to identify. This observation is particularly useful for a practical reconstruction procedure. For a particular case with $\Gamma=\partial B_{R}$, with $B_{R}$ a sufficiently large central ball containing $\Sigma$, we have

Lemma 3.3. Suppose that $\Gamma \subset \partial B_{R}$ and let $\mathbb{H}$ and $\mathbf{M}_{l}$ be defined in (2.32) and (2.41), respectively. Then there holds the following relationship,

$$
\sum_{l=1}^{l_{0}} \mathbf{M}_{l} \mathbb{H}\left(\mathbf{z}_{l}\right) \approx \delta^{-3} R^{3} \overline{\mathbf{D}}_{0}^{-1} \int_{\mathbb{S}} \overline{\mathbf{Q}_{0}(\hat{\mathbf{x}})}\left(\mathbf{H}^{0}-\mathbf{H}_{0}^{0}\right)(\mathbf{x}) d s .
$$

Proof. By straightforward calculations, one can show that $\mathbf{D}_{0}$ is invertible. The proof is completed by directly using (3.13). 


\section{UNique RECOVERY RESUlts FOR MAGNETIC ANOMALY DETECTION}

In this section, by using the asymptotic results obtained in the previous two sections, we establish the major unique recovery results for the geomagnetic detection problem (1.18). To begin with, let $D_{l}^{(1)}$ and $D_{l}^{(2)}, l=1,2, \ldots, l_{0}$, be two sets of magnetic anomalies, which satisfy (2.24) with $\mathbf{z}_{l}$ replaced by $\mathbf{z}_{l}^{(1)}$ and $\mathbf{z}_{l}^{(2)}$, respectively. Correspondingly, the material parameters $\sigma_{l}$ and $\mu_{l}$ are replaced by $\sigma_{l}^{(1)}, \mu_{l}^{(1)}$ and $\sigma_{l}^{(2)}, \mu_{l}^{(2)}$, respectively, for $D_{l}^{(1)}$ and $D_{l}^{(2)}$, $l=1,2, \ldots, l_{0}$. Let $\mathbf{H}_{j}, j=1,2$, be the solutions to (1.12) and (1.17) with $D_{l}$ replaced by $D_{l}^{(1)}$ and $D_{l}^{(2)}$, respectively, and $\mathbf{H}_{j}^{0}$ be the leading-order terms of $\mathbf{H}_{j}$ with respect to $\omega \ll 1$. Denote by $\mathbf{M}_{l}^{(1)}$ and $\mathbf{M}_{l}^{(2)}$ the polarization tensors for $D_{l}^{(1)}$ and $D_{l}^{(2)}$, respectively, $l=1,2, \ldots, l_{0}$. Furthermore, we define

$$
\mathbb{H}_{j}:=\nabla \times \mathcal{A}_{\Sigma_{c}}^{0}\left(-\frac{I}{2}+\mathcal{M}_{\Sigma_{c}}^{0}\right)^{-1}\left[\nu \times\left.\mathbf{H}_{j}^{0}\right|_{\partial \Sigma_{c}}\right], \quad j=1,2 .
$$

We first present the following axillary result.

Lemma 4.1. If the following condition is fulfilled,

$$
\nu \cdot \mathbf{H}_{1}^{0}=\nu \cdot \mathbf{H}_{2}^{0} \neq 0 \text { on } \Gamma,
$$

then there hold that

$$
\sum_{l=1}^{l_{0}} \nabla \Gamma_{0}\left(\mathbf{x}-\mathbf{z}_{l}^{(1)}\right) \mathbf{M}_{l}^{(1)} \mathbb{H}_{1}\left(\mathbf{z}_{l}^{(1)}\right)=\sum_{l=1}^{l_{0}} \nabla \Gamma_{0}\left(\mathbf{x}-\mathbf{z}_{l}^{(2)}\right) \mathbf{M}_{l}^{(2)} \mathbb{H}_{2}\left(\mathbf{z}_{l}^{(2)}\right),
$$

for any $\mathbf{x} \in \mathbb{R}^{3} \backslash \bigcup_{l=1}^{l_{0}}\left(\mathbf{z}_{l}^{(1)} \cup \mathbf{z}_{l}^{(2)}\right)$, and

$$
\mathbb{H}_{1}=\mathbb{H}_{2} \quad \text { in } \quad \mathbb{R}^{3} \backslash \bar{\Sigma}_{c} .
$$

Proof. First, by using the unique continuation principle, one obtains that

$$
\mathbf{H}_{1}^{0}=\mathbf{H}_{2}^{0} \quad \text { in } \quad \mathbb{R}^{3} \backslash \bar{\Sigma},
$$

and in addition that (4.4) holds. Define

$$
\begin{aligned}
\mathbb{T}_{j}(\mathbf{x}):= & \nabla \mathcal{S}_{\Sigma_{c}}^{0}\left(-\frac{I}{2}+\left(\mathcal{K}_{\Sigma_{c}}^{0}\right)^{*}\right)^{-1}\left[\nu \cdot \nabla^{2} \sum_{l=1}^{l_{0}} \Gamma_{0}\left(\cdot-\mathbf{z}_{l}^{(j)}\right) \mathbf{M}_{l}^{(j)} \mathbb{H}_{j}\left(\mathbf{z}_{l}^{(j)}\right)\right](\mathbf{x}) \\
& -\sum_{l=1}^{l_{0}} \nabla^{2} \Gamma_{0}\left(\mathbf{x}-\mathbf{z}_{l}^{(j)}\right) \mathbf{M}_{l}^{(j)} \mathbb{H}_{j}\left(\mathbf{z}_{l}^{(j)}\right), \quad \mathbf{x} \in \mathbb{R}^{3} \backslash \bar{\Sigma}, \quad j=1,2 .
\end{aligned}
$$

By using (3.4) one then has

$$
\mathbb{T}_{1}=\mathbb{T}_{2}, \quad \text { in } \quad \mathbb{R}^{3} \backslash \bar{\Sigma}
$$

Note from (4.5) that $\mathbb{T}_{j}, j=1,2$, are both harmonic functions in $\mathbb{R}^{3} \backslash \bigcup_{l=1}^{l_{0}}\left(\bar{\Sigma}_{c} \cup \mathbf{z}_{l}^{(1)} \cup \mathbf{z}_{l}^{(2)}\right)$. By using the unique continuation again, one obtains

$$
\mathbb{T}_{1}=\mathbb{T}_{2} \quad \text { in } \quad \mathbb{R}^{3} \backslash \bigcup_{l=1}^{l_{0}}\left(\bar{\Sigma}_{c} \cup \mathbf{z}_{l}^{(1)} \cup \mathbf{z}_{l}^{(2)}\right)
$$

By comparing the pole type of terms in $\mathbb{T}_{1}-\mathbb{T}_{2}$ one thus has

$$
\sum_{l=1}^{l_{0}} \nabla \Gamma_{0}\left(\mathbf{x}-\mathbf{z}_{l}^{(1)}\right) \mathbf{M}_{l}^{(1)} \mathbb{H}_{1}\left(\mathbf{z}_{l}^{(1)}\right)=\sum_{l=1}^{l_{0}} \nabla \Gamma_{0}\left(\mathbf{x}-\mathbf{z}_{l}^{(2)}\right) \mathbf{M}_{l}^{(2)} \mathbb{H}_{2}\left(\mathbf{z}_{l}^{(2)}\right) \text { in } \mathbb{R}^{3} \backslash \bigcup_{l=1}^{l_{0}}\left(\bar{\Sigma}_{c} \cup \mathbf{z}_{l}^{(1)} \cup \mathbf{z}_{l}^{(2)}\right) \text {. }
$$

Finally the proof can be completed by using the unique continuation one more time. 
We are ready to present our second main result for the uniqueness in recovering multiple magnetized anomalies.

Theorem 4.1. If there holds

$$
\nu \cdot \mathbf{H}_{1}=\nu \cdot \mathbf{H}_{2} \text { on } \Gamma
$$

with $\mathbf{H}_{1}^{0} \neq 0$ on $\Gamma$ and $\mathbb{H}_{1}$ defined in 4.1) does not vanish in $\mathbf{z}_{l}^{(1)}$, then $\mathbf{z}_{l}^{(1)}=\mathbf{z}_{l}^{(2)}$, $\mu_{l}^{(1)}=\mu_{l}^{(2)}$ and $\sigma_{l}^{(1)}=\sigma_{l}^{(2)}, l=1,2, \ldots, l_{0}$.

Proof. We have (4.3) by using Lemma 4.1. Then by following the proof of Theorem 4.2 in [13] (see also Theorem 7.8 in [2]), one immediately has $\mathbf{z}_{l}^{(1)}=\mathbf{z}_{l}^{(2)}, l=1,2, \ldots, l_{0}$. Next, by using analytic extension, one obtains that

$$
\left.\mathbf{H}_{1}^{0}\right|_{+}=\left.\mathbf{H}_{2}^{0}\right|_{+} \quad \text { on } \quad \partial D_{l} \cup \partial \Sigma_{c}
$$

for $l=1,2, \ldots, l_{0}$. Using the transmission condition across $\partial D_{l}$ (see Lemma 3.3 in [15]), one then has

$$
\left.\nu \cdot \mu_{0} \mathbf{H}_{j}^{0}\right|_{+}=\left.\nu \cdot \mu_{l}^{(j)} \mathbf{H}_{j}^{0}\right|_{-}, j=1,2 \quad \text { on } \quad \partial D_{l},
$$

Let $\varphi_{l, j}^{(0)}, j=1,2$ be the solution to (2.21) with $\mathbf{H}^{0}$ be replaced by $\mathbf{H}_{1}^{0}$ and $\mathbf{H}_{2}^{0}$, respectively. Then by (2.20) and (4.11) one has

$$
\left.\left(1-\frac{\mu_{0}}{\mu_{l}^{(j)}}\right) \nu \cdot \mathbf{H}_{j}^{0}\right|_{+}=\left.\nu \cdot \mathbf{H}_{j}^{0}\right|_{+}-\left.\nu \cdot \mathbf{H}_{j}^{0}\right|_{-}=\varphi_{l, j}^{(0)}, j=1,2 \quad \text { on } \quad \partial D_{l},
$$

Together with (2.30), (4.4) and (4.10), one further has

$$
\left(1-\frac{\mu_{0}}{\mu_{l}^{(1)}}\right)^{-1}\left(\varsigma_{l}^{(1)} I-\left(\mathcal{K}_{\Omega}^{0}\right)^{*}\right)^{-1}\left[\nu \cdot \mathbb{H}_{1}\left(\mathbf{z}_{l}^{(1)}\right)\right]=\left(1-\frac{\mu_{0}}{\mu_{l}^{(2)}}\right)^{-1}\left(\varsigma_{l}^{(2)} I-\left(\mathcal{K}_{\Omega}^{0}\right)^{*}\right)^{-1}\left[\nu \cdot \mathbb{H}_{1}\left(\mathbf{z}_{l}^{(1)}\right)\right],
$$

which implies that

$$
\left(\mu_{l}^{(1)}-\mu_{l}^{(2)}\right)\left(\varsigma_{l}^{(1)} I-\left(\mathcal{K}_{\Omega}^{0}\right)^{*}\right)^{-1}\left(\frac{I}{2}-\left(\mathcal{K}_{\Omega}^{0}\right)^{*}\right)\left(\varsigma_{l}^{(2)} I-\left(\mathcal{K}_{\Omega}^{0}\right)^{*}\right)^{-1}\left[\nu \cdot \mathbb{H}_{1}\left(\mathbf{z}_{l}^{(1)}\right)\right]=0 .
$$

Note that $\int_{\partial \Omega} \nu \cdot \mathbb{H}_{1}\left(\mathbf{z}_{l}^{(1)}\right)=0$ and $\frac{I}{2}-\left(\mathcal{K}_{\Omega}^{0}\right)^{*}$ is invertible on $L_{\diamond}^{2}(\partial \Omega)$, where $L_{\diamond}^{2}(\partial B)$ is the space of functions in $L^{2}$ and has zero average on the boundary, one thus finds from (4.14) that

$$
\left(\mu_{l}^{(1)}-\mu_{l}^{(2)}\right) \nu \cdot \mathbb{H}_{1}\left(\mathbf{z}_{l}^{(1)}\right)=0, \quad l=1,2, \ldots, l_{0} .
$$

Hence $\mu_{l}^{(1)}=\mu_{l}^{(2)}$ by the assumption that $\nu \cdot \mathbb{H}_{1}\left(\mathbf{z}_{l}^{(1)}\right) \neq 0$. By using (2.20) one further has

$$
\mathbf{H}_{j}^{0}=\mathbf{H}_{j}^{0}, j=1,2 \quad \text { in } \quad \mathbb{R}^{3} \backslash \bar{\Sigma}_{c} .
$$

Next we write $\mathbf{H}_{1}$ and $\mathbf{H}_{2}$ in the following form:

$$
\mathbf{H}_{j}=\mathbf{H}_{j}^{0}+\mathbf{H}_{j}^{1} \omega+\mathcal{O}\left(\omega^{2}\right), \quad j=1,2 .
$$

Define $\lambda_{l}^{(j)}:=1 /\left(\mu_{l}^{(j)} \sigma_{l}^{(j)}\right), j=1,2$, then by using (1.17) one derives that

$$
\Delta \mathbf{H}_{j}^{1}(\mathbf{x})-i\left(\lambda_{l}^{(j)}\right)^{-1} \mathbf{H}_{j}^{0}(\mathbf{x})=0, j=1,2, \quad \mathbf{x} \in D_{l},
$$

which together with (4.16) implies that

$$
\Delta\left(\mathbf{H}_{1}^{1}-\mathbf{H}_{2}^{1}\right)(\mathbf{x})=i\left(\left(\lambda_{l}^{(1)}\right)^{-1}-\left(\lambda_{l}^{(2)}\right)^{-1}\right) \mathbf{H}_{1}^{0}(\mathbf{x}), \quad \mathbf{x} \in D_{l},
$$


Furthermore,

$$
\Delta\left(\mathbf{H}_{1}^{1}-\mathbf{H}_{2}^{1}\right)(\mathbf{x})=0, \quad \mathbf{x} \in \Sigma_{s} \backslash \overline{\bigcup_{l=1}^{l_{0}} D_{l}} .
$$

By using the potential theory, one then has

$$
\left(\mathbf{H}_{1}^{1}-\mathbf{H}_{2}^{1}\right)(\mathbf{x})=i\left(\left(\lambda_{l}^{(1)}\right)^{-1}-\left(\lambda_{l}^{(2)}\right)^{-1}\right) \int_{D_{l}} \Gamma_{0}(\mathbf{x}-\mathbf{y}) \mathbf{H}_{1}^{0}(\mathbf{y}) d \mathbf{y}, \quad \mathbf{x} \in D_{l},
$$

Note that $\Delta \mathbf{H}_{1}^{0}=0$ holds in $D_{l}$ and $\mathbf{H}_{1}^{1}-\mathbf{H}_{2}^{1}=0$ holds on $\partial D_{l}$. By following exactly the same strategy as in proof of theorem 2.1 in [19], one can show that

$$
i\left(\left(\lambda_{l}^{(1)}\right)^{-1}-\left(\lambda_{l}^{(2)}\right)^{-1}\right) \mathbf{H}_{1}^{0}(\mathbf{x})=0, \quad \mathbf{x} \in D_{l},
$$

which indicates that $\lambda_{l}^{(1)}=\lambda_{l}^{(2)}$ by the assumption that $\mathbf{H}_{1}^{0}(\mathbf{x})$ does not vanish in $\Gamma$ (so does not vanish in $D_{l}$ ).

The proof is complete.

\section{Concluding Remark}

In this paper, we consider the magnetic anomaly detection using the geomagnetic monitoring. It is formulated as an inverse problem associated with a magnetohydrodynamic system that stems from the widely accepted dynamo theory of geomagnetics. Under certain physically reasonable situation and practical scenario, we establish several uniqueness results in recovering the locations as well as the material parameters of the magnetized anomalies that are presented in the shell of the Earth. The mathematical strategy in establishing the unique recovery results is first based on deriving the static parts of the geomagnetic fields through the low-frequency asymptotic expansions of the geomagnetic fields, and then linearizing the inverse problem by further expanding asymptotically the static fields with respect to the anomaly size. In order to avoid dealing with the nonlinear coupled PDEs in the MHD system, we develop a data continuation strategy into our asymptotic analysis. Nevertheless, we also show how to make use of the properties of the spherical harmonic expansions to relax the severely ill-posed data continuation. We believe the strategy developed here can be extended to dealing with other coupled-physics inverse problems associated with coupled PDEs. This work is a continuation as well as an extension of our recent work [13] on the identification of magnetized anomalies using geomagnetic monitoring, which aims to establish a rigorous mathematical theory for the geomagnetic detection technology. The geomagnetic model considered in [13] is a linear Maxwell system, which is of different mathematical features from the magnetic system in this work.

There are several interesting and challenging topics worth further exploration. First, our theoretical study readily implies an effective reconstruction procedure. In a forthcoming paper, we shall develop efficient numerical reconstruction schemes based on our theoretical results for the magnetic anomaly detection. Second, the magnetic anomalies are presented in the shell of the Earth. The mathematical techniques developed in this work can avoid going inside the core of the Earth. However, if one intends to infer knowledge about the structure of the Earth's core through the generated magnetic field measured outside, one has to deal with the inverse problems associated with the whole MHD system. Finally, the dynamo theory has been used to interpret the magnetic fields of different celestial bodies in addition to that of the Earth. It is interesting to mathematically explore the inference of the interior structures of other planets or stars using the monitoring of their magnetic fields. We note that this technology has been used in space exploration. The mathematical study shall lay out a rigorous theoretical foundation for this kind of technology. 


\section{ACKNOWLEDGMENT}

The work of Y. Deng was supported by NSF grant of China No. 11601528, NSF grant of Hunan No. 2017JJ3432 and No. 2018JJ3619, Innovation-Driven Project of Central South University, No. 2018CX041. The work of H. Liu was supported by the FRG and startup grants from Hong Kong Baptist University, Hong Kong RGC General Research Funds, 12302017 and 12301218.

\section{Appendix A. Proof of Lemma 2.2}

Define

$$
N_{t}:=\left\|\Phi_{s}\right\|_{\mathrm{TH}(\mathrm{div}, \partial \Sigma)}+\left\|\Phi_{c}\right\|_{\mathrm{TH}\left(\mathrm{div}, \partial \Sigma_{\mathrm{c}}\right)}+\left\|\nabla_{\partial \Sigma} \varphi_{s}\right\|_{\mathrm{TH}\left(\mathrm{div}, \partial \Sigma_{\mathrm{c}}\right)} .
$$

To prove Lemma 2.2, we next make use of (2.14)-(2.16) to conduct the asymptotic analysis with respect to $k_{s}$. Denote by $\Delta_{\partial \Sigma}:=\nabla_{\partial \Sigma} \cdot \nabla_{\partial \Sigma}$. First, from (2.13) one can show that

$$
\left\|\Delta_{\partial \Sigma} \varphi_{s}\right\|_{L^{2}(\partial \Sigma)}=\mathcal{O}\left(k_{s}^{2} N_{t}\right) .
$$

Next, we derive the following inequality

$$
\left\|\nabla_{\partial \Sigma} \varphi_{s}\right\|_{\mathrm{TH}(\operatorname{div}, \partial \Sigma)} \leq C\left\|\Delta_{\partial \Sigma} \varphi_{s}\right\|_{L^{2}(\partial \Sigma)},
$$

where $C$ is a constant depends only on $\Sigma$. In fact, for any $\Psi \in L_{T}^{2}(\partial \Sigma)$, by using the Helmholtz decomposition ( [1,7]), there exists a unique $u \in H^{1}(\partial \Sigma), \int_{\partial \Sigma} u d s=0$, such that

$$
\Psi=\nabla_{\partial \Sigma} u+\nabla_{\partial \Sigma} u \times \nu .
$$

By integration by parts there holds

$$
\begin{aligned}
\left\|\nabla_{\partial \Sigma} \varphi_{s}\right\|_{L^{2}(\partial \Sigma)} & =\sup _{\Psi \in L_{T}^{2}(\partial \Sigma)} \frac{\left\langle\nabla_{\partial \Sigma} \varphi_{s}, \Psi\right\rangle}{\|\Psi\|_{\mathrm{TH}(\mathrm{div}, \partial \Sigma)}} \\
& =\sup _{\Psi \in L_{T}^{2}(\partial \Sigma)} \frac{\int_{\partial \Sigma} \nabla_{\partial \Sigma} \varphi_{s} \cdot\left(\nabla_{\partial \Sigma} u+\nabla_{\partial \Sigma} u \times \nu\right) d s}{\left\|\nabla_{\partial \Sigma} u+\nabla_{\partial \Sigma} u \times \nu\right\|_{L^{2}(\partial \Sigma)}} \\
& =\sup _{\Psi \in L_{T}^{2}(\partial \Sigma)} \frac{-\int_{\partial \Sigma}\left(\Delta_{\partial \Sigma} \varphi_{s}\right) u d s}{\left\|\nabla_{\partial \Sigma} u\right\|_{L^{2}(\partial \Sigma)}+\left\|\nabla_{\partial \Sigma} u \times \nu\right\|_{L^{2}(\partial \Sigma)}} \\
& \leq \frac{1}{2} \sup _{\Psi \in L_{T}^{2}(\partial \Sigma)} \frac{\left\|\Delta_{\partial \Sigma} \varphi_{s}\right\|_{L^{2}(\partial \Sigma)}\|u\|_{L^{2}(\partial \Sigma)}}{\left\|\nabla_{\partial \Sigma} u\right\|_{L^{2}(\partial \Sigma)}} \leq C\left\|\Delta_{\partial \Sigma} \varphi_{s}\right\|_{L^{2}(\partial \Sigma)}
\end{aligned}
$$

where the last inequality is obtained by using the Poincaré inequality (see, e.g., [2]). By using (A.1) and (A.2), one thus derives that

$$
\left\|\nabla_{\partial \Sigma} \varphi_{s}\right\|_{\mathrm{TH}(\mathrm{div}, \partial \Sigma)} \sim\left\|\Delta_{\partial \Sigma} \varphi_{s}\right\|_{L^{2}(\partial \Sigma)}=\mathcal{O}\left(k_{s}^{2} N_{t}\right) .
$$

Furthermore, from (2.15) one obtains

$$
\left\|\Phi_{s}\right\|_{\mathrm{TH}(\mathrm{div}, \partial \Sigma)}=\mathcal{O}\left(k_{s}^{2} N_{t}\right) .
$$

By substituting (A.5)-(A.6) into (2.14), there holds

$$
\left(-\frac{I}{2}+\mathcal{M}_{\Sigma_{c}}^{0}\right)\left[\Phi_{c}\right]=\nu \times\left.\mathbf{H}_{0}\right|_{\partial \Sigma_{c}} ^{+}+\mathcal{O}\left(k_{s}^{2}\right) .
$$

Since $-\frac{I}{2}+\mathcal{M}_{\Sigma_{c}}^{0}$ is invertible on $\mathrm{TH}\left(\operatorname{div}, \partial \Sigma_{c}\right)$, by combining (A.5)-(A.7), one can obtain (2.18), which completes the proof. 
Appendix B. Proof of Lemma 2.3

By the continuity of $\nu \times \mathbf{H}$ across $\partial \Sigma$ and $\partial D_{l}$, and using the same notation as that in Appendix A and the jump formulas (2.4) and (2.6), one can show that

$$
\begin{aligned}
& \mathcal{M}_{\Sigma, \Sigma_{c}}^{0}\left[\Psi_{0}\right]+\left(-\frac{I}{2}+\mathcal{M}_{\Sigma}^{0}\right)\left[\Phi_{0}\right]+\mathcal{L}_{\Sigma}^{0}\left[\nabla_{\partial \Sigma} \varphi_{0}\right]+\sum_{l^{\prime}=1}^{l_{0}}\left(\mathcal{M}_{\Sigma, D_{l^{\prime}}}^{0}\left[\Phi_{l^{\prime}}\right]+\mathcal{L}_{\Sigma, D_{l^{\prime}}}^{0}\left[\nabla_{\partial D_{l^{\prime}}} \varphi_{l^{\prime}}\right]\right) \\
= & \mathcal{M}_{\Sigma, \Sigma_{c}}^{k_{s}}\left[\Psi_{0}\right]+\left(\frac{I}{2}+\mathcal{M}_{\Sigma}^{k_{s}}\right)\left[\Phi_{0}\right]+\mathcal{L}_{\Sigma}^{k_{s}}\left[\nabla_{\partial \Sigma} \varphi_{0}\right]+\sum_{l^{\prime}=1}^{l_{0}}\left(\mathcal{M}_{\Sigma, D_{l^{\prime}}}^{k_{s}}\left[\Phi_{l^{\prime}}\right]+\mathcal{L}_{\Sigma, D_{l^{\prime}}}^{k_{s}}\left[\nabla_{\partial D_{l^{\prime}}} \varphi_{l^{\prime}}\right]\right)
\end{aligned}
$$

holds on $\partial \Sigma$ and

$$
\begin{aligned}
& \mathcal{M}_{D_{l}, \Sigma_{c}}^{k_{s}}\left[\Psi_{0}\right]+\mathcal{M}_{D_{l}, \Sigma}^{k_{s}}\left[\Phi_{0}\right]+\mathcal{L}_{D_{l}, \Sigma}^{k_{s}}\left[\nabla_{\partial \Sigma} \varphi_{0}\right]-\frac{\Phi_{l}}{2}+\sum_{l^{\prime}=1}^{l_{0}}\left(\mathcal{M}_{D_{l}, D_{l^{\prime}}}^{k_{s}}\left[\Phi_{l^{\prime}}\right]+\mathcal{L}_{D_{l}, D_{l^{\prime}}}^{k_{s}}\left[\nabla_{\partial D_{l^{\prime}}} \varphi_{l^{\prime}}\right]\right) \\
= & \mathcal{M}_{D_{l}, \Sigma_{c}}^{k_{l}}\left[\Psi_{0}\right]+\mathcal{M}_{D_{l}, \Sigma}^{k_{l}}\left[\Phi_{0}\right]+\mathcal{L}_{D_{l}, \Sigma}^{k_{l}}\left[\nabla_{\partial \Sigma} \varphi_{0}\right]+\frac{\Phi_{l}}{2}+\sum_{l^{\prime}=1}^{l_{0}}\left(\mathcal{M}_{D_{l}, D_{l^{\prime}}}^{k_{l}}\left[\Phi_{l^{\prime}}\right]+\mathcal{L}_{D_{l}, D_{l^{\prime}}}^{k_{l}}\left[\nabla_{\partial D_{l^{\prime}}} \varphi_{l^{\prime}}\right]\right),
\end{aligned}
$$

holds on $\partial D_{l}, l=1,2, \ldots, l_{0}$. On the other hand, by using the continuity of $\nu \cdot \mu \mathbf{H}$ across $\partial \Sigma$ and $\partial D_{l}$ (see Lemma 3.3 in [15]), one can further show that

$$
\begin{aligned}
& \mathcal{N}_{\Sigma, \Sigma_{c}}^{0}\left[\Psi_{0}\right]+\mathcal{N}_{\Sigma}^{0}\left[\Phi_{0}\right]+\left(\frac{I}{2}+\left(\mathcal{K}_{\Sigma}^{0}\right)^{*}\right)\left[\Delta_{\partial \Sigma} \varphi_{0}\right]+\sum_{l^{\prime}=1}^{l_{0}}\left(\mathcal{N}_{\Sigma, D_{l^{\prime}}}^{0}\left[\Phi_{l^{\prime}}\right]+\mathcal{K}_{\Sigma, D_{l^{\prime}}}^{0}\left[\varphi_{l^{\prime}}\right]\right) \\
= & \mathcal{N}_{\Sigma, \Sigma_{c}}^{k_{s}}\left[\Psi_{0}\right]+\mathcal{N}_{\Sigma}^{k_{s}}\left[\Phi_{0}\right]+\left(-\frac{I}{2}+\left(\mathcal{K}_{\Sigma}^{k_{s}}\right)^{*}\right)\left[\Delta_{\partial \Sigma} \varphi_{0}\right]+k_{s}^{2} \nu \cdot \mathcal{A}_{\Sigma}^{k_{s}}\left[\nabla_{\partial \Sigma} \varphi_{0}\right] \\
& +\sum_{l^{\prime}=1}^{l_{0}}\left(\mathcal{N}_{\Sigma, D_{l^{\prime}}}^{k_{s}}\left[\Phi_{l^{\prime}}\right]+\mathcal{K}_{\Sigma, D_{l^{\prime}}}^{k_{s}}\left[\varphi_{l^{\prime}}\right]\right),
\end{aligned}
$$

holds on $\partial \Sigma$ and

$$
\begin{aligned}
& \mu_{0}\left(\mathcal{N}_{D_{l}, \Sigma_{c}}^{k_{s}}\left[\Psi_{0}\right]+\mathcal{N}_{D_{l}, \Sigma}^{k_{s}}\left[\Phi_{0}\right]+\mathcal{K}_{D_{l}, \Sigma}^{k_{s}}\left[\Delta_{\partial \Sigma} \varphi_{0}\right]+\frac{\varphi_{l}}{2}\right. \\
& \left.+\sum_{l^{\prime}=1}^{l_{0}}\left(\mathcal{N}_{D_{l}, D_{l^{\prime}}}^{k_{s}}\left[\Phi_{l^{\prime}}\right]+k_{s}^{2} \nu \cdot \mathcal{A}_{D_{l}, D_{l^{\prime}}}^{k_{s}}\left[\nabla_{\partial D_{l^{\prime}}} \varphi_{l^{\prime}}\right]+\mathcal{K}_{D_{l}, D_{l^{\prime}}}^{k_{s}}\left[\Delta_{\partial D_{l^{\prime}}} \varphi_{l^{\prime}}\right]\right)\right) \\
= & \mu_{l}\left(\mathcal{N}_{D_{l}, \Sigma_{c}}^{k_{l}}\left[\Psi_{0}\right]+\mathcal{N}_{D_{l}, \Sigma}^{k_{l}}\left[\Phi_{0}\right]+\mathcal{K}_{D_{l}, \Sigma}^{k_{l}}\left[\Delta_{\partial \Sigma} \varphi_{0}\right]-\frac{\varphi_{l}}{2}\right. \\
& \left.+\sum_{l^{\prime}=1}^{l_{0}}\left(\mathcal{N}_{D_{l}, D_{l^{\prime}}}^{k_{l}}\left[\Phi_{l^{\prime}}\right]+k_{l}^{2} \nu \cdot \mathcal{A}_{D_{l}, D_{l^{\prime}}}^{k_{l}}\left[\nabla_{\partial D_{l^{\prime}}} \varphi_{l^{\prime}}\right]+\mathcal{K}_{D_{l}, D_{l^{\prime}}}^{k_{l}}\left[\Delta_{D_{l^{\prime}}} \varphi_{l^{\prime}}\right]\right)\right),
\end{aligned}
$$

holds on $\partial D_{l}$, where $\mathcal{K}_{\Sigma^{\prime}, \Sigma^{\prime \prime}}^{k}: L^{2}\left(\partial \Sigma^{\prime}\right) \rightarrow L^{2}\left(\partial \Sigma^{\prime \prime}\right), \Sigma^{\prime}, \Sigma^{\prime \prime} \in\left\{\Sigma, \Sigma_{c}, D_{1}, D_{2}, \ldots, D_{l_{0}}\right\}, k \in$ $\left\{0, k_{s}, k_{1}, k_{2}, \ldots, k_{l_{0}}\right\}$, are defined by

$$
\mathcal{K}_{\Sigma^{\prime}, \Sigma^{\prime \prime}}^{k}[\varphi]:=\left.\nu \cdot \nabla \mathcal{S}_{\Sigma^{\prime \prime}}^{k}[\varphi]\right|_{\partial \Sigma^{\prime}} ^{-}
$$

If $\Sigma^{\prime}=\Sigma^{\prime \prime}$, then $\mathcal{K}_{\Sigma^{\prime}, \Sigma^{\prime}}^{k}[\varphi]:=\left(\mathcal{K}_{\Sigma^{\prime}}^{k}\right)^{*}$. Similarly, $\mathcal{A}_{\Sigma^{\prime}, \Sigma^{\prime \prime}}^{k}: \mathrm{TH}\left(\operatorname{div}, \partial \Sigma^{\prime}\right) \rightarrow \mathrm{TH}\left(\operatorname{div}, \partial \Sigma^{\prime \prime}\right)$, is defined by

$$
\mathcal{A}_{\Sigma^{\prime}, \Sigma^{\prime \prime}}^{k}[\Phi]:=\left.\mathcal{A}_{\Sigma^{\prime \prime}}^{k}[\Phi]\right|_{\partial \Sigma^{\prime}} ^{-}
$$


Furthermore, by using the boundary magnetic field $\nu \times \mathbf{H}$ on $\partial \Sigma_{c}$, one also has

$$
\begin{aligned}
& \left(-\frac{I}{2}+\mathcal{M}_{\Sigma_{c}}^{k_{s}}\right)\left[\Psi_{0}\right]+\mathcal{M}_{\Sigma_{c}, \Sigma}^{k_{s}}\left[\Phi_{0}\right]+\mathcal{L}_{\Sigma_{c}, \Sigma}^{k_{s}}\left[\nabla_{\partial \Sigma} \varphi_{0}\right] \\
& +\sum_{l^{\prime}=1}^{l_{0}}\left(\mathcal{M}_{\Sigma_{c}, D_{l^{\prime}}}^{k_{s}}\left[\Phi_{l^{\prime}}\right]+\mathcal{L}_{\Sigma_{c}, D_{l^{\prime}}}^{k_{s}}\left[\nabla_{\partial D_{l^{\prime}}} \varphi_{l^{\prime}}\right]\right)=\nu \times \mathbf{H} . \quad \text { on } \partial \Sigma_{c}
\end{aligned}
$$

By combining (B.2)-(B.7), along with straightforward calculations and the similar inequality that is deduced in Appendix A, one can derive the following estimates sequentially,

$$
\left\|\Phi_{0}\right\|_{\mathrm{TH}(\operatorname{div}, \partial \Sigma)}=\mathcal{O}\left(\omega N_{f}\right), \quad\left\|\nabla_{\partial \Sigma} \varphi_{0}\right\|_{\mathrm{TH}(\operatorname{div}, \partial \Sigma)}=\mathcal{O}\left(\omega N_{f}\right), \quad\left\|\Phi_{l}\right\|_{\mathrm{TH}\left(\operatorname{div}, \partial D_{l}\right)}=\mathcal{O}\left(\omega N_{f}\right),
$$

where $l=1,2, \ldots, l_{0}$ and $N_{f}:=\mathcal{O}\left(\left\|\Psi_{0}\right\|_{\mathrm{TH}\left(\operatorname{div}, \partial \Sigma_{c}\right)}+\sum_{l^{\prime}=1}^{l_{0}}\left\|\nabla_{\partial D_{l^{\prime}}} \varphi_{l^{\prime}}\right\|_{\mathrm{TH}\left(\operatorname{div},\left(\partial D_{l}\right)\right.}\right)$, and

$$
\begin{aligned}
\Psi_{0}= & \left(-\frac{I}{2}+\mathcal{M}_{\Sigma_{c}}^{0}\right)^{-1}[\nu \times \mathbf{H}]-\sum_{l^{\prime}=1}^{l_{0}}\left(-\frac{I}{2}+\mathcal{M}_{\Sigma_{c}}^{0}\right)^{-1} \mathcal{L}_{\Sigma_{c}, D_{l^{\prime}}}^{0}\left[\Delta_{D_{l^{\prime}}} \varphi_{l^{\prime}}\right] \\
& +\mathcal{O}\left(\omega \sum_{l^{\prime}=1}^{l_{0}}\left\|\Delta_{D_{l^{\prime}}} \varphi_{l^{\prime}}\right\|_{L^{2}\left(\partial D_{l}\right)}\right) .
\end{aligned}
$$

Hence

$$
\begin{aligned}
& \left({ }_{\varsigma} I-\left(\mathcal{K}_{D_{l}}^{0}\right)^{*}+\mathcal{P}_{D_{l}, \Sigma_{c}} \mathcal{L}_{\Sigma_{c}, D_{l}}^{0}\right)\left[\Delta_{\partial D_{l}} \varphi_{l}\right]-\sum_{l^{\prime} \neq l}^{l_{0}}\left(\mathcal{K}_{D_{l}, D_{l^{\prime}}}^{0}-\mathcal{P}_{D_{l}, \Sigma_{c}} \mathcal{L}_{\Sigma_{c}, D_{l^{\prime}}}^{0}\right)\left[\Delta_{\partial D_{l^{\prime}}} \varphi_{l^{\prime}}\right] \\
= & \mathcal{P}_{D_{l}, \Sigma_{c}}\left[\nu \times\left.\mathbf{H}\right|_{\partial \Sigma_{c}}\right]+\mathcal{O}(\omega),
\end{aligned}
$$

where the operators $\mathcal{P}_{D_{l}, \Sigma_{c}}: \mathrm{TH}\left(\operatorname{div}, \partial \Sigma_{c}\right) \rightarrow \mathrm{TH}\left(\operatorname{div}, \partial D_{l}\right), l=1,2, \ldots, l_{0}$, are defined by

$$
\mathcal{P}_{D_{l}, \Sigma_{c}}[\Phi]:=\mathcal{N}_{D_{l}, \Sigma_{c}}^{0}\left(-\frac{I}{2}+\mathcal{M}_{\Sigma_{c}}^{0}\right)^{-1}[\Phi] .
$$

Noting that $\left(\mathcal{K}_{D_{l}}^{0}\right)^{*}$ and $\mathcal{P}_{D_{l}, \Sigma_{c}} \mathcal{L}_{\Sigma_{c}, D_{l}}^{0}$ are compact operators on $L^{2}\left(\partial D_{l}\right)$, one can prove the invertibility of $\varsigma_{l} I-\left(\mathcal{K}_{D_{l}}^{0}\right)^{*}+\mathcal{P}_{D_{l}, \Sigma_{c}} \mathcal{L}_{\Sigma_{c}, D_{l}}^{0}$ on $L^{2}\left(\partial D_{l}\right)$ by following a similar proof of Lemma 2.2 in [13. In fact, by the Fredholm theory, it suffices to show the uniqueness of a trivial solution to the following integral equation,

$$
\left(\varsigma_{l} I-\left(\mathcal{K}_{D_{l}}^{0}\right)^{*}+\mathcal{P}_{D_{l}, \Sigma_{c}} \mathcal{L}_{\Sigma_{c}, D_{l}}^{0}\right)\left[\Delta_{\partial D_{l}} \varphi_{l}\right]=0 .
$$

Note that there exists only a trial solution to the following system (see Appendix A in [13])

$$
\begin{cases}\nabla \times \mathbf{H}=0, \quad \nabla \cdot \mathbf{H}=0, & \text { in }\left(\mathbb{R}^{3} \backslash \overline{D_{l} \cup \Sigma_{c}}\right) \cup D_{l}, \\ \nu_{l} \times\left.\mathbf{H}\right|_{+}=\nu_{l} \times\left.\mathbf{H}\right|_{-}, & \text {on } \partial D_{l}, \\ \left.\mu_{0} \nu_{l} \cdot \mathbf{H}\right|_{+}=\left.\mu_{l} \nu_{l} \cdot \mathbf{H}\right|_{-}, & \text {on } \partial D_{l}, \\ \nu \times\left.\mathbf{H}\right|_{+}=0,\left.\quad \int_{\partial \Sigma_{c}} \nu \cdot \mathbf{H}\right|_{+}=0, & \text { on } \partial \Sigma_{c} \\ \mathbf{H}(\mathbf{x})=\mathcal{O}\left(\|\mathbf{x}\|^{-2}\right), \quad\|\mathbf{x}\| \rightarrow \infty . & \end{cases}
$$

On the other hand, one can verify that

$$
\mathbf{H}=\left(-\nabla \mathcal{S}_{D_{l}}^{0}+\nabla \times \mathcal{A}_{\Sigma_{c}}^{0}\left(-\frac{I}{2}+\mathcal{M}_{\Sigma_{c}}^{0}\right)^{-1} \nu \times \nabla \mathcal{S}_{D_{l}}^{0}\right)\left[\Delta_{\partial D_{l}} \varphi_{l}\right]
$$


is also the solution to (B.13). Therefore, one has

$$
\left(-\nabla \mathcal{S}_{D_{l}}^{0}+\nabla \times \mathcal{A}_{\Sigma_{c}}^{0}\left(-\frac{I}{2}+\mathcal{M}_{\Sigma_{c}}^{0}\right)^{-1} \nu \times \nabla \mathcal{S}_{D_{l}}^{0}\right)\left[\Delta_{\partial D_{l}} \varphi_{l}\right]=0 \quad \text { in } \mathbb{R}^{3} \backslash \overline{\Sigma_{c}} .
$$

Hence $\Delta_{\partial D_{l}} \varphi_{l}=0$, which proves the unique trial solution to (B.12). Note that $D_{l}, l=$ $1,2, \ldots, l_{0}$ are small inclusions which are disjoint from each, one can prove the unique solvability of (B.10) (see Appendix B in [13]).

\section{ApPENDix C. HARMONIC REPRESENTATION OF VECTORIAL SPHERICAL POLYNOMIALS}

In this appendix, we shall represent $\mathbf{A}_{n}^{m}(\hat{\mathbf{x}}) \xi$, where $\mathbf{A}_{n}^{m}(\hat{\mathbf{x}})$ is defined in (3.10) and $\xi \in \mathbb{R}^{3}$, in terms of vectorial spherical harmonic functions. Recall that the vectorial spherical harmonic functions of degree $n$ are composed of $\mathbf{M}_{n+1}^{m}(\hat{\mathbf{x}}), \mathbf{Q}_{n-1}^{m}(\hat{\mathbf{x}})$ and $\mathbf{T}_{n}^{m}(\hat{\mathbf{x}})$, which are defined in (3.2) and (3.16). From (3.10) one has

$$
\begin{aligned}
\mathbf{A}_{n}^{m}(\hat{\mathbf{x}}) \xi= & (n+1) \hat{\mathbf{x}} \nabla_{S} Y_{n}^{m}(\hat{\mathbf{x}})^{T} \xi+(n+1) Y_{n}^{m}(\hat{\mathbf{x}}) \xi-(n+1)(n+3) Y_{n}^{m}(\hat{\mathbf{x}}) \hat{\mathbf{x}} \hat{\mathbf{x}}^{T} \xi \\
& -\nabla_{S}\left(\nabla_{S} Y_{n}^{m}(\hat{\mathbf{x}})^{T} \xi\right)+(n+2) \nabla_{S} Y_{n}^{m}(\hat{\mathbf{x}}) \hat{\mathbf{x}}^{T} \xi,
\end{aligned}
$$

By vector calculus identity and integration by parts, there holds

$$
\begin{aligned}
\int_{\mathbb{S}} \overline{\mathbf{T}_{n}^{m}(\hat{\mathbf{x}})} \cdot\left(\mathbf{A}_{n}^{m}(\hat{\mathbf{x}}) \xi\right) d s & =(n+1) \int_{\mathbb{S}} Y_{n}^{m}(\hat{\mathbf{x}}) \overline{\mathbf{T}_{n}^{m}(\hat{\mathbf{x}})} \cdot \xi d s \\
& =(n+1) \int_{\mathbb{S}}\|\mathbf{x}\|^{n} Y_{n}^{m}(\hat{\mathbf{x}}) \hat{\mathbf{x}} \cdot\left(\xi \times \nabla\left(\|\mathbf{x}\|^{n} \overline{Y_{n}^{m}(\hat{\mathbf{x}})}\right)\right) d s \\
& =(n+1) \int_{B_{1}} \nabla \cdot\left(\|\mathbf{x}\|^{n} Y_{n}^{m}(\hat{\mathbf{x}}) \xi \times \nabla\left(\|\mathbf{x}\|^{n} \overline{Y_{n}^{m}(\hat{\mathbf{x}})}\right)\right) d \mathbf{x} \\
& =(n+1) \int_{B_{1}} \nabla\left(\|\mathbf{x}\|^{n} \overline{Y_{n}^{m}(\hat{\mathbf{x}})}\right) \cdot\left(\nabla\left(\|\mathbf{x}\|^{n} Y_{n}^{m}(\hat{\mathbf{x}})\right) \times \xi\right) d \mathbf{x}=0
\end{aligned}
$$

Hence, $\mathbf{A}_{n}^{m}(\hat{\mathbf{x}}) \xi$ is a linear combination of the spherical harmonics $\left\{\mathbf{N}_{n+1}^{m}(\hat{\mathbf{x}})\right\}$ and $\left\{\mathbf{Q}_{n-1}^{m}(\hat{\mathbf{x}})\right\}$. By straightforward computations, one can obtain that

$$
\begin{aligned}
& \int_{\mathbb{S}} \overline{\mathbf{N}_{n^{\prime}+1}^{m^{\prime}}(\hat{\mathbf{x}})} \cdot\left(\hat{\mathbf{x}} \nabla_{S} Y_{n}^{m}(\hat{\mathbf{x}})^{T} \xi\right) d s=\frac{n^{\prime}+1}{n^{\prime}} \int_{\mathbb{S}} \overline{\mathbf{Q}_{n^{\prime}-1}^{m^{\prime}}(\hat{\mathbf{x}})} \cdot\left(\hat{\mathbf{x}} \nabla_{S} Y_{n}^{m}(\hat{\mathbf{x}})^{T} \xi\right) d s \\
= & \left(n^{\prime}+1\right) \int_{\mathbb{S}} \overline{Y_{n^{\prime}}^{m^{\prime}(\hat{\mathbf{x}})}} \nabla_{S} Y_{n}^{m}(\hat{\mathbf{x}})^{T} \xi d s .
\end{aligned}
$$

Similarly, one can show that

$$
\begin{aligned}
& \int_{\mathbb{S}} \overline{\mathbf{N}_{n^{\prime}+1}^{m^{\prime}}(\hat{\mathbf{x}})} \cdot\left(Y_{n}^{m}(\hat{\mathbf{x}}) \xi\right) d s \\
= & -\int_{\mathbb{S}} Y_{n}^{m}(\hat{\mathbf{x}}) \nabla_{S}{\overline{Y_{n^{\prime}}^{m^{\prime}}(\hat{\mathbf{x}})}}^{T} \xi d s+\left(n^{\prime}+1\right) \int_{\mathbb{S}} \overline{Y_{n^{\prime}}^{m^{\prime}}(\hat{\mathbf{x}})} Y_{n}^{m}(\hat{\mathbf{x}}) \hat{\mathbf{x}}^{T} \xi d s,
\end{aligned}
$$

and

$$
\begin{aligned}
& \int_{\mathbb{S}} \overline{\mathbf{Q}_{n^{\prime}-1}^{m^{\prime}}(\hat{\mathbf{x}})} \cdot\left(Y_{n}^{m}(\hat{\mathbf{x}}) \xi\right) d s \\
= & \int_{\mathbb{S}} Y_{n}^{m}(\hat{\mathbf{x}}) \nabla_{S}{\overline{Y_{n^{\prime}}^{m^{\prime}}(\hat{\mathbf{x}})}}^{T} \xi d s+n^{\prime} \int_{\mathbb{S}} \overline{Y_{n^{\prime}}^{m^{\prime}}(\hat{\mathbf{x}})} Y_{n}^{m}(\hat{\mathbf{x}}) \hat{\mathbf{x}}^{T} \xi d s,
\end{aligned}
$$

and

$$
\begin{aligned}
& \int_{\mathbb{S}} \overline{\mathbf{N}_{n^{\prime}+1}^{m^{\prime}}(\hat{\mathbf{x}})} \cdot\left(Y_{n}^{m}(\hat{\mathbf{x}}) \hat{\mathbf{x}} \hat{\mathbf{x}}^{T} \xi\right) d s=\frac{n^{\prime}+1}{n^{\prime}} \int_{\mathbb{S}} \overline{\mathbf{Q}_{n^{\prime}-1}^{m^{\prime}}(\hat{\mathbf{x}})} \cdot\left(Y_{n}^{m}(\hat{\mathbf{x}}) \hat{\mathbf{x}} \hat{\mathbf{x}}^{T} \xi\right) d s \\
= & \left(n^{\prime}+1\right) \int_{\mathbb{S}} \overline{Y_{n^{\prime}}^{m^{\prime}}(\hat{\mathbf{x}})} Y_{n}^{m}(\hat{\mathbf{x}}) \hat{\mathbf{x}}^{T} \xi d s .
\end{aligned}
$$


Next, for the last two terms in (C.1), using integration by parts, we have

$$
\begin{aligned}
& \int_{\mathbb{S}} \overline{\mathbf{N}_{n^{\prime}+1}^{m^{\prime}}(\hat{\mathbf{x}})} \cdot \nabla_{S}\left(\nabla_{S} Y_{n}^{m}(\hat{\mathbf{x}})^{T} \xi\right) d s=-\int_{\mathbb{S}} \overline{\mathbf{Q}_{n^{\prime}-1}^{m^{\prime}}(\hat{\mathbf{x}})} \cdot \nabla_{S}\left(\nabla_{S} Y_{n}^{m}(\hat{\mathbf{x}})^{T} \xi\right) d s \\
= & -\int_{\mathbb{S}} \nabla_{S} \overline{Y_{n^{\prime}}^{m^{\prime}}(\hat{\mathbf{x}})} \cdot \nabla_{S}\left(\nabla_{S} Y_{n}^{m}(\hat{\mathbf{x}})^{T} \xi\right) d s \\
= & \int_{\mathbb{S}} \Delta_{S} \overline{Y_{n^{\prime}}^{m^{\prime}}(\hat{\mathbf{x}})} \nabla_{S} Y_{n}^{m}(\hat{\mathbf{x}})^{T} \xi d s=-n^{\prime}\left(n^{\prime}+1\right) \int_{\mathbb{S}} \overline{Y_{n^{\prime}}^{m^{\prime}}(\hat{\mathbf{x}})} \nabla_{S} Y_{n}^{m}(\hat{\mathbf{x}})^{T} \xi d s .
\end{aligned}
$$

Furthermore, there holds

$$
\begin{aligned}
& \int_{\mathbb{S}} \overline{\mathbf{N}_{n^{\prime}+1}^{m^{\prime}}(\hat{\mathbf{x}})} \cdot\left(\nabla_{S} Y_{n}^{m}(\hat{\mathbf{x}}) \hat{\mathbf{x}}^{T} \xi\right) d s=-\int_{\mathbb{S}} \overline{\mathbf{Q}_{n^{\prime}-1}^{m^{\prime}}(\hat{\mathbf{x}})} \cdot\left(\nabla_{S} Y_{n}^{m}(\hat{\mathbf{x}}) \hat{\mathbf{x}}^{T} \xi\right) d s \\
= & -\int_{\mathbb{S}} \nabla_{S} \overline{Y_{n^{\prime}}^{m^{\prime}(\hat{\mathbf{x}})}} \cdot\left(\nabla_{S} Y_{n}^{m}(\hat{\mathbf{x}}) \hat{\mathbf{x}}^{T} \xi\right) d s \\
= & -\int_{\mathbb{S}} \nabla_{S} \overline{Y_{n^{\prime}}^{m^{\prime}}(\hat{\mathbf{x}})} \cdot\left(\nabla\left(Y_{n}^{m}(\hat{\mathbf{x}}) \hat{\mathbf{x}}^{T} \xi\right)-Y_{n}^{m}(\hat{\mathbf{x}}) \xi\right) d s \\
= & -n^{\prime}\left(n^{\prime}+1\right) \int_{\mathbb{S}} \overline{Y_{n^{\prime}}^{m^{\prime}}(\hat{\mathbf{x}})} Y_{n}^{m}(\hat{\mathbf{x}}) \hat{\mathbf{x}}^{T} \xi d s+\int_{\mathbb{S}} Y_{n}^{m}(\hat{\mathbf{x}}) \nabla_{S} \overline{Y_{n^{\prime}}^{m^{\prime}}(\hat{\mathbf{x}})}
\end{aligned}
$$

In the following, we define

$$
\mathbf{a}_{n^{\prime}, n}^{m^{\prime}, m}:=\int_{\mathbb{S}} \overline{Y_{n^{\prime}}^{m^{\prime}}(\hat{\mathbf{x}})} \nabla_{S} Y_{n}^{m}(\hat{\mathbf{x}}) d s, \quad \mathbf{b}_{n^{\prime}, n}^{m^{\prime}, m}:=\int_{\mathbb{S}} \overline{Y_{n^{\prime}}^{m^{\prime}}(\hat{\mathbf{x}})} Y_{n}^{m}(\hat{\mathbf{x}}) \hat{\mathbf{x}} d s .
$$

By combining (C.3)-(C.8) and using (‥1), one thus has

$$
\begin{aligned}
& \int_{\mathbb{S}} \overline{\mathbf{N}_{n^{\prime}+1}^{m^{\prime}}(\hat{\mathbf{x}})} \cdot\left(\mathbf{A}_{n}^{m}(\hat{\mathbf{x}}) \xi\right) d s \\
= & \left(\left(n^{\prime}+1\right)\left(n^{\prime}+n+1\right) \mathbf{a}_{n^{\prime}, n}^{m^{\prime}, m}-\left(n^{\prime}+1\right)\left(n^{\prime}+n+1\right)(n+2) \mathbf{b}_{n^{\prime}, n}^{m^{\prime}, m}+\overline{\mathbf{a}_{n, n^{\prime}}^{m, m^{\prime}}}\right)^{T} \xi
\end{aligned}
$$

and

$$
\int_{\mathbb{S}} \overline{\mathbf{Q}_{n^{\prime}+1}^{m^{\prime}}(\hat{\mathbf{x}})} \cdot\left(\mathbf{A}_{n}^{m}(\hat{\mathbf{x}}) \xi\right) d s=\left(n^{\prime}\left(n-n^{\prime}\right) \mathbf{a}_{n^{\prime}, n}^{m^{\prime}, m}-n^{\prime}\left(n-n^{\prime}\right)(n+2) \mathbf{b}_{n^{\prime}, n}^{m^{\prime}, m}-\overline{\mathbf{a}_{n, n^{\prime}}^{m, m^{\prime}}}\right)^{T} \xi .
$$

By using the following elementary result (cf. [20])

$$
\mathbf{a}_{n^{\prime}, n}^{m^{\prime}, m}=\mathbf{b}_{n^{\prime}, n}^{m^{\prime}, m}=0, \quad \text { for any } n^{\prime} \neq n-1, n+1, \text { and } m^{\prime} \neq m-1, m, m+1,
$$

one finally obtains

$$
\begin{aligned}
\mathbf{A}_{n}^{m}(\hat{\mathbf{x}}) \xi= & \sum_{m^{\prime}=m-1}^{m+1}\left(\left(\mathbf{c}_{n-1, n}^{m^{\prime}, m}\right)^{T} \xi \mathbf{N}_{n}^{m^{\prime}}(\hat{\mathbf{x}})+\left(\mathbf{c}_{n+1, n}^{m^{\prime}, m}\right)^{T} \xi \mathbf{N}_{n+2}^{m^{\prime}}(\hat{\mathbf{x}})\right. \\
& \left.+\left(\mathbf{d}_{n-1, n}^{m^{\prime}, m}\right)^{T} \xi \mathbf{Q}_{n-2}^{m^{\prime}}(\hat{\mathbf{x}})+\left(\mathbf{d}_{n+1, n}^{m^{\prime}, m}\right)^{T} \xi \mathbf{Q}_{n}^{m^{\prime}}(\hat{\mathbf{x}})\right),
\end{aligned}
$$

where

$$
\mathbf{c}_{n^{\prime}, n}^{m^{\prime}, m}:=\frac{\left(n^{\prime}+1\right)\left(n^{\prime}+n+1\right) \mathbf{a}_{n^{\prime}, n}^{m^{\prime}, m}-\left(n^{\prime}+1\right)\left(n^{\prime}+n+1\right)(n+2) \mathbf{b}_{n^{\prime}, n}^{m^{\prime}, m}+\overline{\mathbf{a}_{n, n^{\prime}}^{m, m^{\prime}}}}{\left(n^{\prime}+1\right)\left(2 n^{\prime}+1\right)},
$$

and

$$
\mathbf{d}_{n^{\prime}, n}^{m^{\prime}, m}:=\frac{n^{\prime}\left(n-n^{\prime}\right) \mathbf{a}_{n^{\prime}, n}^{m^{\prime}, m}-n^{\prime}\left(n-n^{\prime}\right)(n+2) \mathbf{b}_{n^{\prime}, n}^{m^{\prime}, m}-\overline{\mathbf{a}_{n, n^{\prime}}^{m, m^{\prime}}}}{n^{\prime}\left(2 n^{\prime}+1\right)} .
$$




\section{REFERENCES}

[1] H. Ammari, Y. Deng, P. Millien, Surface plasmon resonance of nanoparticles and applications in imaging, Arch. Ration. Mech. Anal., 220 (2016), 109-153.

[2] H. Ammari, H. Kang, Polarization and Moment Tensors With Applications to Inverse Problems and Effective Medium Theory, Applied Mathematical Sciences, Springer-Verlag, Berlin Heidelberg, 2007.

[3] H. Ammari, H. Kang, H. Lee, M. Lim, and S. Yu, Enhancement of near cloaking for the full Maxwell equations, SIAM J. Appl. Math., 73 (2013), no. 6, 2055-2076.

[4] H. Ammari and J.-C. Nédélec, Low-frequency electromagnetic scattering, SIAM J. Math. Anal., 31 (2000), no. 4, 836-861.

[5] H. Ammari, M.S. Vogelius and D. Volkov, Asymptotic formulas for perturbations in the electromagnetic fields due to the presence of inhomogeneities of small diameter. II. The full Maxwell equations., J. Math. Pures Appl. (9), 80 (2001), no. 8, 769-814.

[6] G. Backus, R. Parker, and C. Constable, Foundations of Geomagnetism, Cambridge University Press, 1996.

[7] A. Buffa, M. Costabel, and D. Sheen, On traces for $H(\operatorname{curl}, \Omega)$ in lipschitz domains. J. Math. Anal. Appl., 276 (2002), 845-867.

[8] K. H. Chan, K. Zhang and J. Zou, Spherical interface dynamos: mathematical theory, finite element approximation, and application, SIAM J. Numer. Anal., 44 (2006), 1877-1902.

[9] K.Chan, K.Zhang, X.Liao G. Schubert and J. Zou, A three-dimensional spherical nonlinear interface dynamo, The Astrophysical Journal, 596 (2003), 663-679.

[10] Y. He and J. Zou, A priori estimates and optimal finite element approximation of the MHD flow in smooth domains, ESAIM: Math. Model. Numer. Anal., 52 (2018), 181-206.

[11] G. Dassios, Low-Frequency Scattering, Chapter 1.5.1 in Scattering: Scattering and Inverse Scattering in Pure and Applied Science, R. Pike and P. Sabatier, eds., Academic Press (2002), 230-244.

[12] Y. Deng, H. Li and H. Liu, On spectral properties of Neuman-Poincaré operator on spheres and plasmonic resonances in $3 D$ elastostatics, Journal of Spectral Theory, in press.

[13] Y. Deng, J. Li, H. Liu, On identifying magnetized anomalies using geomagnetic monitoring, Arch. Ration. Mech. Anal., 231 (2019), no. 1, 153-187.

[14] Y. Deng, H. Liu and X. Liu, Recovery of an embedded obstacle and the surrounding medium for Maxwell's system, arXiv:1801.02008.

[15] Y. Deng, H. Liu and G. Uhlmann, On an inverse boundary problem arising in brain imaging, arXiv:1702.00154.

[16] R. Hollerbach and C. A. Jones, A geodynamo model incorporating a finitely conducting inner core, Phys. Earth Planet. Inter., 75 (1993), 317-327.

[17] R. E. Kleinman, Low Frequency Electromagnetic Scattering, Chapter 1 in Electromagnetic Scattering, P. L.E. Uslenghi, eds., Academic Press (1978), 1-28.

[18] S. Labrosse, Thermal and magnetic evolution of the Earth's core, Phys. Earth Planet. Inter., 140 (2003), $127-143$.

[19] H. Liu and G. Uhmann, Determing both sound speed and internal source in thermo- and photo-acoutic tomography, Inverse Problems, 31 2015, 105005.

[20] J. C. Nédélec, Acoustic and Electromagnetic Equations: Integral Representations for Harmonic Problems, Springer-Verlag, New York, 2001.

[21] P. H. Roberts and G. A. Glatzmaier, The geodynamo, past, present and future, Geophys. Astrophys. Fluid Dyn., 94 (2001), 47-84.

[22] A. Sakuraba and M. Kono, Effect of the inner core on the numerical solution of the magnetodydrodynamic dynamo, Phys. Earth Planet. Inter., 111 (1999), 105-121.

[23] R. H. Torres, Maxwell's equations and dielectric obstacles with Lipschitz boundaries, J. London Math. Soc. (2) 57 (1998), 157-169.

[24] N. Weiss, Dynamos in planets, stars and galaxies, Astronomy and Geophysics, 43 (2002), 3.09-3.15.

[25] Dynamo theory, https://en.wikipedia.org/wiki/Dynamo_theory.

[26] X. Zhan, K. Zhang and R. Zhu, A full-sphere convection-driven dynamo: Implications for the ancient geomagnetic field, Physics of the Earth and Planetary Interiors, 187 (2011), 328-335.

[27] K. Zhang, G. Schubert, Magnetohydrodynamics in rapidly rotating spherical systems, Ann. Rev. Fluid Mech., 32 (2000), 409-443. 
School of Mathematics and Statistics, Central South University, Changsha, Hunan, China.

E-mail address: youjundeng@csu.edu.cn, dengyijun_001@163.com

School of Science, Qilu University of Technology (Shandong Academy of Sciences), Jinan, Shandong, China

E-mail address: lijinhong@qlu.edu.cn

Department of Mathematics, Hong Kong Baptist University, Kowloon, Hong Kong SAR, CHINA

E-mail address: hongyu.liuip@gmail.com, hongyuliu@hkbu.edu.hk 